\title{
An Empirical Study into Comparing Conventional and Islamic Banks in the UAE
}

\author{
Manoj Kapur \\ DBA Research Scholar \\ SP Jain School of Global Management, Mumbai, India \\ E-mail: manoj.m.kapur@gmail.com
}

Received: July 17, 2020

Accepted: July 30, 2020

Online Published: August 17, 2020

doi: I0.4628I/ijafr.v5i2.7I6

URL: https://doi.org/I0.4628I/ijafr.v5i2.7I6

\begin{abstract}
Banking system constitutes the fundamental pillar of every economy. Banks acts financial intermediaries between sectors that have excess funds and those that are in deficit. Islamic banks operate under Sharia principles of risk sharing and interest prohibition as contrasted with conventional banks that buy capital to pool funds and sell capital to generate interest income or profit. This paper applies banks' internal factors related to their balance sheet and income statement and using a total of 23 financial ratios pertaining to the internal factors, it attempts to compare and contrast between conventional and Islamic banks. This research explains the structure, operation and management of banks in the UAE coupled with the functioning of Islamic banks. The paper also aims to determine the profitable and efficient banks among the chosen sample. The sample includes 12 banks, equally distributed between Islamic and conventional banks using data between the periods of 20I4 - 20I8. The sample is broadly categorized based on profitability ratio, efficiency ratio, asset indicator ratio and risk ratios. Correlation and Regression analysis is used to determine a substantial ratio analysis between conventional and Islamic banks. Results from the study reveal indicators of financial characteristics such as profitability ratios, efficiency ratios, asset quality indicators and risk/ risk management ratios. The results clarify that Islamic banks are operationally efficient and profitable because of risks sharing and greater dependency on deposits capital. However, on an overall basis, the ratios indicate conventional banks have higher scores than their counterparts.
\end{abstract}

Keywords: Islamic Banking; Conventional Banking; Interest-Free; Profit-And-Loss Sharing; Financial Ratios.

\section{JEL Classification Codes: F37.}

\section{Introduction}

The history of banking in the GCC region dates back to 1918 with the establishment of the first bank in Bahrain. The regional banking sector is unique in nature owing to oil wealth and lending business that focuses on building, real estate, customer loans and heavy external from competition protection. Dubai Islamic Bank was the first standalone Islamic Bank established in 1975 in the UAE. Over the years, several international banks have also established Islamic banking division that upholds traditional Islamic values and offers products and services compliant with Sharia principles. Today, more than 200 Islamic banks operate in 70 countries with $\$ 2.6$ billion in assets under management. In its initial years, Islamic banks witnessed its growth in South Asia and GCC countries. Islamic banks receive funds from deposits instead of shareholders. In contrast to the conventional banks, the principle of risk sharing lead to a better return on equity for Islamic banks. Statistical evidence shows that Islamic banks tend to achieve a higher profit margin compared to conventional banks.

Over the years, the banking system globally has evolved in its offerings to suit the changing consumer demands. One of the primary determinants of this change resulted from the religious beliefs of the people resulting in the phenomenal growth of Islamic Banking System. The predominance of these banks is in countries with significant Muslim population such as Iran, Pakistan, and Sudan but not restricted to them. The difference between the Islamic Bank and the Conventional Bank is generally the framework and principle that governs them. Sharia or Islamic law governs Islamic banking institutions. The holy book of Islam, Quran prohibits all transactions from receiving and paying interest, Riba, as it is believed that interest bearing credit structure leads to an inequitable distribution of societal income. To overcome this challenge, Islamic banks earns revenue from profit sharing agreements. The concept of interest is restructured with a time markup of payments and commercial financing commissions under the Islamic banking model. 
The research paper aims to compare the conventional banks and Islamic banks using several parametersfor the years 20I4-2018. It will include measuring the performance of the selected banks on the basis of different ratio calculation viz profitability, efficiency, asset quality, liquidity and liquidity risk management. While there are several literatures available under each head, it is very rare that these have been considered together for research purposes. This paper applies banks' internal factors related to their balance sheet and income statement and using a total of 23 financial ratios pertaining to the internal factors, the paper attempts to compare and contrast between conventional and Islamic banks. This research explains the structure, operation and management of banks in the UAE region coupled with the functioning of Islamic banks. The paper also aims to determine the profitable and efficient banks among the chosen sample.

\section{I.I Literature Review}

Bashier (1983) investigated on the regulatory compliance among Islamic and Non-Islamic banks in Bangladesh. The study involved 223 perceptions among 23 tradition banks and seven Islamic banks between 2003 to 2013. The outcome of the study ensured that Islamic banks having higher cash flow as compared to their traditional counterparts were better productive.

Coats and Fant (1993) examined and analyzed the financial distress prediction using neural network approach applying financial ratios. In the similar context, Al-Hassan, Khamis, and Oulidi (2010) analyzed the gradual evolution of the banking sector in the Gulf Cooperation Council (GCC) member countries. The study dealt with cross-border linkages, balance sheet exposures and risks, concentration, ownership, financial soundness and recent trends in credit growth. The research identified the variety of risks that posed a threat to the financial stability of the banking sector in the context of the global crisis and suggested ways to mitigate them.

Demirgue-Kunt and Hizinga (1999) using data for 80 countries spanning a period between 1988-1995 showed that differences in interest bank profitability and margins reflected several determinants namely bank macroeconomic conditions, overall financial structure, explicit and implicit bank taxation, deposit insurance regulation, and many regulatory and institutional indicators. The results of the paper indicated that a greater bank asset to GDP ratio and a lower market concentration ratio lead to lower margins and profits.

Hussain, Islam, Gunasekaran and Maskooki (2002) pointed out that recently, economies and financial institutions in the GCC and in particular UAE sought to create closer ties among each other. This called for better coordination of accounting regulations resulting in improved cooperation and improved the efficiency of the financial institutions among these countries.

Al-Tamimi and Al-Amiri (2003) conducted research on the differences between conventional and Islamic banks within the middle east based on financial ratios. Islamic banks primarily work on the principles of risk sharing interest prohibition. Yet each bank dealt with similar challenges and thus the study could not conclusively prove whether financial ratios help can truly differentiate between the two categories of banks. Their research used 26 financial ratios and applied logic, neural network and k-means to find whether researchers and regulators can use these ratios in providing specific differences between these two sets of banks.

Islam (2003) analyzed the internal performance of domestic and foreign banks in Bahrain, Oman, the United Arab Emirates by studying their financial ratios. The study showed that the commercial banks in these countries are well geared through the adoption of modern banking services, they have improved their performance in the past few years. Most banks were financially sound as per international standards and financial ratios benchmarks.

Essayyad and Madani (2003) investigated the concentration, efficiency, and profitability of commercial banks in Saudi Arabia. Applying the techniques of regression analysis, the authors investigated the determinants of Saudi bank efficiency, concentration, and profitability. The importance of the study stemmed from the theory that highly concentrated banking or credit market brought in inefficiencies that can potentially hinder the bank's access to credit leading slowing down growth.

Rosly and Bakar (2003) studied the performance of Islamic banks and conventional (mainstream) banks in Malaysia using key ratios which included return on assets, asset quality and investment ratios. The study results indicated that Islamic banks had higher returns on asset as the overhead cost of managing Islamic banks were lower. However, higher return on asset did not signify that Islamic banks were more efficient than conventional banks. Conventional banks were considered efficient due to scale and its economies even though Islamic banks had differentiated interest like products.

Hume (2004) investigated the possibility to distinguish between conventional and Islamic banks in the Gulf Cooperation Council (GCC) based on budgetary qualities. Similarly, Iqbal (2006) analyzed the varied interpretations of riba and the lack of consensus in its meaning. The study concluded that riba in barter loans also falls in the category of real riba and takes effect when any of the six commodities namely gold, silver, wheat, barley, dates and salt are exchanged with a time lag in different measures. This mainly attributed to the possibility of underlying commodity price change creating uncertainty for both parties of the exchange.

Grais and Pellegrini (2006) reviewed corporate governance challenges of institutions that offered Islamic financial services (IIFS) and suggested options that addressed them. The paper suggested a distinctive treatment of corporate Governance issues for IIFS when compared to conventional corporate governance. The paper suggested a governance model based on a 
combination of internal and external arrangements and that relies heavily on transparency and disclosure of information that is market relevant.

Al-Ajmi, Hussain, and Al-Saleh (2009) investigated into the intentions of Bahraini customers in choosing a bank, their understanding of bank products and their relative benefits. Considered to be the first study that samples three distinctive set of clients, namely Islamic, conventional and both. Using questionnaire method to 1000 clients and applying Mann-Whitney and Kruskal-Wallis tests and factor analysis the study concluded that Islamic religious belief, social responsibility and cost benefit are the primary determinants in bank selection among Bahraini bank clients. The study also revealed that though the clients of conventional and Islamic banks share common requirements, they do differ in their product understandings. Islamic bank clients are more familiar with sharia compliant products/services.

Al-Muharrami and Matthews (2009) evaluated the performance of the GCC banking industry in the pretext of the "Structure-Conduct-Performance" hypothesis using sample data between the period of 1993-2002 and applied panel estimation distinguishing between bank fixed effects and country fixed effects. The paper examined the "Relative-Market-Power and the Efficient-Structure hypotheses" distinguishing between the two by applying a non-parametric measure of technical efficiency, and found that the banking industry in the GCC countries is best explained by the mainstream SCP hypothesis.

Despite minor differences, UAE shares a common economic, cultural and political similarity with other GCC countries with an efficient, stable and profitable collective banking system (Al-Muharrami \& Matthews, 2009). The banking sector's contributes to GDP is next to oil and gas sector. The region grew rapidly during the period 2000-2008 primarily from oil exports. The Islamic banking and financial services industry have shown rapid growth over the past ten years, reaching a milestone of over $\$ 2$ trillion in value by 20I5. GCC banks have grown strong, well-capitalized and modern banking services have been adopted. Islamic banks accounted for $18 \%$ of the financial system in 2007 that accounted for around $36 \%$ of global Islamic financial assets. Over the last decade, Islamic Banks has grown at a rate of 20 - 30 percent per year, which is three times higher than conventional banks. The Islamic banking system in comparison with traditional banks was less affected by the economic and financial crisis since investments in toxic assets and derivatives are strictly prohibited (Iqbal, 2006).

Merchant (2012) studied the performance of Islamic and conventional banks during different time periods including crises and post crises periods. While there were many researches conducted to uphold that Islamic banks have much better viability of generating profits and are considered stable, the research objectives included the measure taken by these banks to curb the ill effects of the crises. The methodology involved sampling I7 banks in the GCC during the period 2008 to 201 I and used CAMEL test factors. The results of the 2 -tailed test showed that Islamic banks were able to significantly increase their loan loss reserves and equity to total asset ratio. Islamic banks also had adequate capital structures with lower return on average equity. Islamic banks and conventional banks were comparable in terms of asset quality and liquidity.

Hanif, Tariq, and Tahir (2012) analyzed and compared the performance of Islamic and conventional banks in Pakistan on the basis of their internal and external factors. They used nine ratios to ascertain profitability, liquidity and credit risk which were part of the internal factors. The external factors were specific to analyzing the customer preferences and perception while banking with Islamic or conventional banks. The results of the research indicated that conventional banks were better in managing profitability and liquidity while Islamic banks had a head-start in capital maintenance and liquidity risk management. Key findings included the perception of sharia compliance for Islamic banks variety of products for conventional banks which were considered as motivating factors for each type of banks.

Beck, Demirgüç-Kunt and Merrouche (2010) stated that products offered by the conventional banks can be converted to sharia compliant products by making minor changes. The research utilized anecdotal evidences to prove that Islamic banks can be cost effective than conventional banks with insignificant differences in business orientation, efficiency, asset quality and stability. The study also revealed that countries where conventional banks enjoyed higher market share, it was found that Islamic banks had lower level of stability but were cost effective. Additionally, the research highlighted that Islamic banks had performed better in the 2008 financial crises as they enjoyed higher capitalization and liquidity coverage as compared to the conventional banks.

Based on the above literature review, it is clearly evident that in order to judge the banks image, one has to look beyond regular normal ratios like ROA, ROE, etc. The approach has to be more granular that can consider efficiency, profitability, risk and risk management into more detail. Considering these issues, the research is done using 23 ratios covering several areas of the banks financial statement. These ratios may not be touched upon using traditional ratio analysis approach. The main headers would include the profitability ratios, efficiency ratios, asset quality ratios, risk ratios and risk management ratios which would be sub divided into 23 further ratios.

The objective of the study is to ascertain if these ratios significantly impact the dependent variables of the conventional and Islamic banks in the UAE.

\section{I.2 Hypotheses of the Study}

In order to examine the research objectives, it is imperative to compare the financial ratios of conventional and Islamic banks. For these purposes we have defined the following research hypothesis: 
Hypotheses I:There is no significant difference between profitability ratios of conventional banks and Islamic banks in the UAE.

Hypothesis 2: There is no significant difference between efficiency ratios of the conventional banks and Islamic banks in the UAE.

Hypothesis 3: There is no significant difference between asset quality of the conventional banks and Islamic banks in the UAE.

Hypothesis 4: There is no significant difference between Liquidity of the conventional and Islamic banks in the UAE

Hypothesis 5: There is no significant difference between liquidity risk management of the conventional and Islamic banks in the UAE.

The alternative hypothesis for all the above would include a significant difference in the financial ratios being tested at each stage.

\section{Data and Methodology}

The purpose of the study is to compare the conventional and Islamic banks that have been licensed in the UAE. The study will aid to channelize the future development of both these banking sectors in terms of deposits, profitability and efficiency. The proposed study will analytical in nature and will use secondary data from the annual published financial statements of the conventional and Islamic banks in the UAE. The authors have chosen quantitative analysis using excel to arrive at the conclusion. Data relating to profitability, efficiency, asset quality, liquidity and risk management has been taken from the annual reports since 2014 to 20I8. The sample size consists of top 6 conventional banks and Islamic banks, respectively, operating in the UAE. These include:

Conventional banks:

- First Abu Dhabi Bank (FAB),

- Emirates NBD (ENBD),

- Abu Dhabi Commercial Bank (ADCB),

- Mashreq Bank (MB),

- Commercial Bank Dubai (CBD)

- Ras Al Kaimah Bank (RAK)

Islamic Banks:

- Abu Dhabi Islamic Bank (ADIB),

- Dubai Islamic Bank (DIB),

- Noor Bank (NB) and

- Sharjah Islamic Bank (SIB)

- Al Hilal Bank (AHB)

- Emirates Islamic Bank (EIB)

2.I Table I-Dependent and Independent Variables

\begin{tabular}{|c|c|c|c|c|c|c|}
\hline Sr No & Ratio & variables & variables & Formula & $\begin{array}{l}\text { Dependent/ } \\
\text { Independent }\end{array}$ & Description \\
\hline $\mathrm{I}$ & $\begin{array}{l}\text { Bank } \\
\text { profitability } \\
\text { ratios }\end{array}$ & $\mathrm{ROA}$ & $\begin{array}{l}\text { Return on } \\
\text { Assets }\end{array}$ & $\begin{array}{lr}\text { net } & \text { income/ } \\
\text { average } & \text { total } \\
\text { assets. } & \\
\end{array}$ & Dependent & $\begin{array}{l}\text { The return on assets shows the } \\
\text { percentage of how profitable a } \\
\text { company's assets are in } \\
\text { generating revenue. }\end{array}$ \\
\hline 2 & $\begin{array}{l}\text { Bank } \\
\text { efficiency } \\
\text { ratios }\end{array}$ & NIM & $\begin{array}{l}\text { Net Interest } \\
\text { Margin }\end{array}$ & $\begin{array}{l}\text { (net interest } \\
\text { income-net } \\
\text { interest expenses) } \\
/ \text { average total } \\
\text { assets. }\end{array}$ & Dependent & $\begin{array}{l}\text { Net interest margin is a } \\
\text { measure of the difference } \\
\text { between the interest income } \\
\text { generated by banks or other } \\
\text { financial institutions and the } \\
\text { amount of interest paid out to } \\
\text { their lenders, relative to the } \\
\text { amount of their assets. }\end{array}$ \\
\hline
\end{tabular}




\begin{tabular}{|c|c|c|c|c|}
\hline 3 & $\begin{array}{l}\text { Asset-quality PEA } \\
\text { indicators }\end{array}$ & $\begin{array}{l}\text { Provision to } \\
\text { Earning } \\
\text { Assets }\end{array}$ & $\begin{array}{l}\text { provision for loan } \\
\text { losses / average } \\
\text { total loans and } \\
\text { advances. }\end{array}$ & Dependent \\
\hline
\end{tabular}

A loan loss provision is an expense set aside as an allowance for uncollected loans and loan payments. This provision is used to cover a number of factors associated with potential loan losses, including bad loans, customer defaults, and renegotiated terms of a loan that incur lower than previously estimated payments

\begin{tabular}{|c|c|c|c|c|}
\hline 4 & $\begin{array}{l}\text { Liquidity } \\
\text { ratios }\end{array}$ & CTA & $\begin{array}{l}\text { Cash } \\
\text { toAssets }\end{array}$ & $\begin{array}{l}\text { cash / average Dependent } \\
\text { total assets. }\end{array}$ \\
\hline
\end{tabular}

A liquid asset requirement, or ratio, is defined as the obligation of commercial banks to maintain a predetermined percentage of total deposits and certain other liabilities in the form of liquid assets.

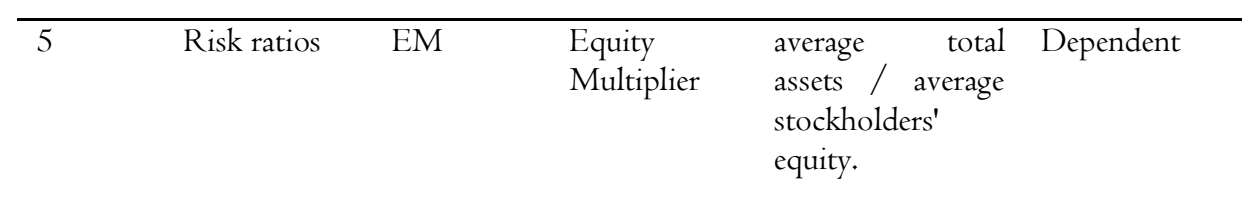
The equity multiplier is a financial leverage ratio that measures the amount of a bank's assets that are financed by its shareholders by comparing total assets with total shareholder's equity. In other words, the equity multiplier shows the percentage of assets that are financed or owed by the shareholders.

\begin{tabular}{lllll}
\hline 6 & Bank & ROE & Return on net income/ Independent \\
profitability & & Equity & $\begin{array}{l}\text { average } \\
\text { stockholders' } \\
\end{array}$ & \\
& & equity.
\end{tabular}
the return on equity is a measure of the profitability of a business in relation to the equity, also known as net assets or assets minus liabilities. ROE is a measure of how well a company uses investments to generate earnings growth

\begin{tabular}{lllll}
\hline 7 & Bank & PM & Profit & net income/ Independent \\
profitability & & Margin & operating income. & \\
ratios & & & &
\end{tabular}

Profit margin, net margin, net profit margin or net profit ratio is a measure of profitability. It is calculated by finding the net profit as a percentage of the revenue.

\begin{tabular}{|c|c|c|c|c|c|c|}
\hline 8 & $\begin{array}{l}\text { Bank } \\
\text { profitability } \\
\text { ratios }\end{array}$ & ROD & $\begin{array}{l}\text { Return on } \\
\text { Deposits }\end{array}$ & $\begin{array}{lr}\text { net income/ } \\
\text { average } & \text { total } \\
\text { customer } & \\
\text { deposits. } & \\
\end{array}$ & Independent & $\begin{array}{l}\text { It is calculated by dividing net } \\
\text { income to average total } \\
\text { customer deposits }\end{array}$ \\
\hline 9 & $\begin{array}{l}\text { Bank } \\
\text { profitability } \\
\text { ratios }\end{array}$ & NOM & $\begin{array}{l}\text { Net } \\
\text { Operating } \\
\text { Margin }\end{array}$ & $\begin{array}{l}\text { operating profit } \\
\text { or income/ } \\
\text { interest income. }\end{array}$ & Independent & $\begin{array}{l}\text { The net interest margin in } \\
\text { banking is similar to the gross } \\
\text { profit margin for operating } \\
\text { companies. It is equal to a } \\
\text { bank's total interest income }\end{array}$ \\
\hline
\end{tabular}




\begin{tabular}{|c|c|c|c|c|c|c|}
\hline & & & & & & minus total interest expense \\
\hline $\mathrm{IO}$ & $\begin{array}{l}\text { Bank } \\
\text { efficiency } \\
\text { ratios }\end{array}$ & IEE & $\begin{array}{l}\text { Interest } \\
\text { Income to } \\
\text { Expenses }\end{array}$ & $\begin{array}{l}\text { (interest } \\
\text { income-interest } \\
\text { expenses) } \\
\text { average total } \\
\text { loans } \\
\text { advances. }\end{array}$ & Independent & $\begin{array}{l}\text { It is calculated by dividing } \\
\text { NIM to average total loans } \\
\text { and advances }\end{array}$ \\
\hline II & $\begin{array}{l}\text { Bank } \\
\text { efficiency } \\
\text { ratios } \\
\end{array}$ & OEA & $\begin{array}{l}\text { Operating } \\
\text { Expense to } \\
\text { Assets }\end{array}$ & $\begin{array}{l}\text { operating } \\
\text { expenses/ average } \\
\text { total assets. }\end{array}$ & Independent & $\begin{array}{l}\text { It is calculated by dividing } \\
\text { operating expenses to average } \\
\text { total assets }\end{array}$ \\
\hline $\mathrm{I2}$ & $\begin{array}{l}\text { Bank } \\
\text { efficiency } \\
\text { ratios }\end{array}$ & OIA & $\begin{array}{l}\text { Operating } \\
\text { Income to } \\
\text { Assets }\end{array}$ & $\begin{array}{l}\text { operating } \\
\text { income/ average } \\
\text { total assets. }\end{array}$ & Independent & $\begin{array}{l}\text { It is calculated by dividing } \\
\text { operating income to average } \\
\text { total assets }\end{array}$ \\
\hline $\mathrm{I3}$ & $\begin{array}{l}\text { Bank } \\
\text { efficiency } \\
\text { ratios }\end{array}$ & $\overline{\text { OER }}$ & $\begin{array}{l}\text { Operating } \\
\text { Expenses to } \\
\text { Revenue }\end{array}$ & $\begin{array}{l}\text { operating } \\
\text { expenses/ } \\
\text { operating income } \\
\text { (revenue). }\end{array}$ & Independent & $\begin{array}{l}\text { Expense to income ratio is } \\
\text { calculated by dividing the } \\
\text { operating expenses by the } \\
\text { operating income generated } \\
\text { i.e.net interest income plus the } \\
\text { other income. Cost-to-income } \\
\text { ratio is important for } \\
\text { determining the profitability } \\
\text { of a bank }\end{array}$ \\
\hline $\mathrm{I} 4$ & $\begin{array}{l}\text { Bank } \\
\text { efficiency } \\
\text { ratios }\end{array}$ & ATO & $\begin{array}{l}\text { Asset } \\
\text { Turnover }\end{array}$ & $\begin{array}{lr}\text { interest } & \text { income/ } \\
\text { average } & \text { total } \\
\text { assets. } & \end{array}$ & Independent & $\begin{array}{l}\text { Asset Turnover measures how } \\
\text { quickly a company turns over } \\
\text { its asset through sales. It is } \\
\text { calculated as Revenue divided } \\
\text { by Total Assets }\end{array}$ \\
\hline I5 & $\begin{array}{l}\text { Bank } \\
\text { efficiency } \\
\text { ratios }\end{array}$ & NNIM & $\begin{array}{l}\text { Net Non- } \\
\text { Interest } \\
\text { Margin }\end{array}$ & $\begin{array}{l}\text { (net non-interest } \\
\text { income-net non- } \\
\text { interest expenses) } \\
\text { /average total } \\
\text { assets. }\end{array}$ & Independent & $\begin{array}{l}\text { No interest margin is a } \\
\text { financial measurement that } \\
\text { helps asses the usefulness of } \\
\text { revenue from non-interest } \\
\text { items such as fees and service } \\
\text { charges. }\end{array}$ \\
\hline 16 & $\begin{array}{l}\text { Asset-quality } \\
\text { indicators }\end{array}$ & LR & Loan Ratio & $\begin{array}{lr}\text { average } & \text { total } \\
\text { loans } & \text { and } \\
\text { advances } & / \\
\text { average } & \text { total } \\
\text { assets. } & \\
\end{array}$ & Independent & $\begin{array}{l}\text { It is calculated by dividing } \\
\text { Average total loans to average } \\
\text { total assets }\end{array}$ \\
\hline I7 & $\begin{array}{l}\text { Asset-quality } \\
\text { indicators }\end{array}$ & LTD & $\begin{array}{ll}\text { Loans } & \text { to } \\
\text { Deposits } & \end{array}$ & $\begin{array}{lr}\text { average } & \text { total } \\
\text { loans } & \text { and } \\
\text { advances } & / \\
\text { average } & \text { total } \\
\text { customer } & \\
\text { deposits. } & \\
\end{array}$ & Independent & $\begin{array}{l}\text { The loan-to-deposit ratio } \\
\text { (LDR) is used to assess a } \\
\text { bank's liquidity by comparing } \\
\text { a bank's total loans to its total } \\
\text { deposits for the same period. }\end{array}$ \\
\hline 18 & $\begin{array}{l}\text { Liquidity } \\
\text { ratios }\end{array}$ & CTD & $\begin{array}{ll}\text { Cash to } \\
\text { Deposits }\end{array}$ & $\begin{array}{l}\text { cash / average } \\
\text { total customer } \\
\text { deposits. }\end{array}$ & Independent & $\begin{array}{l}\text { Cash Deposit ratio (CDR) is } \\
\text { the ratio of how much a bank } \\
\text { lends out of the deposits it has } \\
\text { mobilized. It indicates how } \\
\text { much of a bank's core funds } \\
\text { are being used for lending, the } \\
\text { main banking activity }\end{array}$ \\
\hline 19 & Risk ratios & DTA & $\begin{array}{l}\text { Deposits to } \\
\text { Assets }\end{array}$ & $\begin{array}{l}\text { average total } \\
\text { customer deposits } \\
/ \quad \text { average total } \\
\text { assets. }\end{array}$ & Independent & $\begin{array}{l}\text { Deposits to Assets is a ratio } \\
\text { that tells you that to what } \\
\text { extent bank's assets have been } \\
\text { funded from a stable source }\end{array}$ \\
\hline
\end{tabular}




\begin{tabular}{|c|c|c|c|c|c|c|}
\hline 20 & Risk ratios & ETD & $\begin{array}{l}\text { Equity } \\
\text { toDeposits }\end{array}$ & $\begin{array}{l}\text { average } \\
\text { shareholders' } \\
\text { equity / average } \\
\text { customer total } \\
\text { deposits. }\end{array}$ & Independent & $\begin{array}{l}\text { It is calculated by dividing } \\
\text { average shareholders' equity to } \\
\text { average customer total } \\
\text { deposits. }\end{array}$ \\
\hline $2 \mathrm{I}$ & Risk ratios & TLE & $\begin{array}{l}\text { Total } \\
\text { Liabilities to } \\
\text { Equity }\end{array}$ & $\begin{array}{l}\text { average total } \\
\text { liabilities } \\
\text { average } \\
\text { stockholders' } \\
\text { equity. }\end{array}$ & Independent & $\begin{array}{l}\text { The Debt to Equity ratio is } \\
\text { calculated as total liabilities } \\
\text { divided by total shareholders' } \\
\text { equity }\end{array}$ \\
\hline 22 & Risk ratios & TLSC & $\begin{array}{l}\text { Total } \\
\text { Liabilities to } \\
\text { Shareholder } \\
\text { Capital }\end{array}$ & $\begin{array}{l}\text { average total } \\
\text { liabilities } \\
\text { shareholder } \\
\text { contributed } \\
\text { capital. }\end{array}$ & Independent & $\begin{array}{l}\text { It is calculated by dividing } \\
\text { Average total loans to average } \\
\text { total assets }\end{array}$ \\
\hline 23 & Risk ratios & RETA & $\begin{array}{l}\text { Retained } \\
\text { Earnings to } \\
\text { Total Assets }\end{array}$ & $\begin{array}{l}\text { retained earnings } \\
/ \quad \text { average total } \\
\text { assets." }\end{array}$ & Independent & $\begin{array}{l}\text { The retained earnings to total } \\
\text { assets ratio measures the } \\
\text { banks' ability to accumulate } \\
\text { earnings using its assets }\end{array}$ \\
\hline
\end{tabular}

\subsection{Descriptive Analysis}

The study is proposed to include regression analysis to ascertain underlying factors that contribute to the performance of conventional and Islamic banks in the UAE. Based on the formation of hypothesis, five different models have been established. These models will encompass one independent variable and several dependent variables within each hypothesis. This has been described in Table 2 below. Mehta and Bhavani (2017) stated that there are several internal and external variables that are determinants of the profitability of the profitability of the banks in the UAE. Most notable among these were the cost efficiency ratio, adequate capital adequacy ratio and improvement in the asset quality ratio. However, it is also important to keep in mind Return on Assets which could also enhance profitability by diversifying income sources.

\subsection{Accounting Ratios}

Across the UAE, conventional banks have adopted accounting policies based on International Accounting Standards Board (IASB)and IFRS standards (Hussain et al., 2002). In contrast, Islamic banks follow accounting policies established by the Accounting and Auditing Organization for Islamic Financial Institutions (AAOFI). Each Islamic bank establishes a Sharia committee that monitors and examines all bank transactions in compliance with Islamic principles. The ratios under study are categorized based on profit, efficiency, quality of assets and risk. Existing research focused solely on profitability and efficiency. This research uses ratios such as Bank Return on Asset (ROA), Profit Margin (PM), Deposit Return (ROA), Equity Return (ROE), Return on Shareholder Capital (ROSC) and various other ratios for analysis purposes. Based on the previous study, that Islamic banks are more moneymaking than conventional banks. Provision for earning profit (PEA), show how a bank manages its assets. High PEA implies higher reserves for bad loans and unforeseen emergencies and to lower risk.

\subsection{Table 2-Descriptive Statistics for conventional and Islamic banks based on defined hypothesis}

\begin{tabular}{|c|c|c|c|c|c|c|c|c|c|c|c|c|}
\hline \multirow[t]{2}{*}{ Sr. No } & \multirow[t]{2}{*}{ Ratios } & \multirow{2}{*}{$\begin{array}{l}\text { Variables } \\
\text { Dependent/ } \\
\text { Independent }\end{array}$} & \multirow{2}{*}{$\begin{array}{c}\text { Sample } \\
\text { Size }\end{array}$} & \multicolumn{4}{|c|}{ Conventional Banks } & \multicolumn{5}{|c|}{ Islamic Banks } \\
\hline & & & & $\begin{array}{l}\text { Min } \\
\text { value }\end{array}$ & $\begin{array}{l}\text { Max } \\
\text { value }\end{array}$ & Mean & $\begin{array}{l}\text { Standard } \\
\text { Deviation }\end{array}$ & $\begin{array}{c}\text { Sample } \\
\text { Size }\end{array}$ & $\begin{array}{l}\text { Min } \\
\text { value }\end{array}$ & $\begin{array}{l}\text { Max } \\
\text { value }\end{array}$ & Mean & $\begin{array}{l}\text { Standard } \\
\text { Deviation }\end{array}$ \\
\hline \multicolumn{13}{|c|}{ Bank profitability ratios } \\
\hline $\mathrm{I}$ & $\begin{array}{l}\text { return on } \\
\text { assets } \\
(\mathrm{ROA})\end{array}$ & Dependent & 30 & 0.0137 & 0.0418 & 0.0198 & 0.0063 & 30 & 0.0018 & 0.0256 & 0.0142 & 0.0060 \\
\hline 2 & $\begin{array}{l}\text { return on } \\
\text { equity } \\
\text { (ROE) }\end{array}$ & Independent & 30 & 0.0875 & 0.2040 & 0.1349 & 0.0286 & 30 & 0.0158 & 0.2072 & 0.1088 & 0.0434 \\
\hline 3 & $\begin{array}{l}\text { profit } \\
\text { margin } \\
(\mathrm{PM})\end{array}$ & Independent & 30 & 0.1727 & $0.639 \mathrm{I}$ & 0.4529 & 0.1265 & 30 & 0.0423 & 0.6696 & $0.378 I$ & 0.1482 \\
\hline
\end{tabular}




\begin{tabular}{|c|c|c|c|c|c|c|c|c|c|c|c|c|}
\hline 4 & $\begin{array}{l}\text { return on } \\
\text { deposits } \\
\text { (ROD) }\end{array}$ & Independent & 30 & 0.0199 & $0.639 \mathrm{I}$ & $0.030 \mathrm{I}$ & $0.009 \mathrm{I}$ & 30 & 0.0026 & 0.0349 & 0.0200 & 0.0082 \\
\hline 5 & $\begin{array}{l}\text { net } \\
\text { operating } \\
\text { margin } \\
(\mathrm{NOM})\end{array}$ & Independent & 30 & 0.7929 & $\mathrm{I} .452 \mathrm{I}$ & 1.0978 & 0.1482 & 30 & 0.7347 & 1.5003 & I.I557 & 0.1770 \\
\hline \multicolumn{13}{|c|}{ Bank efficiency ratios } \\
\hline 6 & $\begin{array}{l}\text { net interest } \\
\text { margin } \\
(\mathrm{NIM})\end{array}$ & Dependent & 30 & 0.0170 & 0.0792 & 0.0327 & 0.0160 & 30 & $0.011 \mathrm{I}$ & 0.0352 & 0.0245 & 0.0075 \\
\hline 7 & $\begin{array}{l}\text { operating } \\
\text { expense to } \\
\text { assets } \\
\text { (OEA) }\end{array}$ & Independent & 30 & 0.0066 & 0.0432 & 0.0164 & 0.0092 & 30 & 0.0104 & $0.02 \mathrm{II}$ & 0.0158 & 0.0032 \\
\hline 8 & $\begin{array}{l}\text { operating } \\
\text { income to } \\
\text { assets } \\
\text { (OIA) }\end{array}$ & Independent & 30 & 0.0245 & $0.102 \mathrm{I}$ & 0.0472 & 0.0203 & 30 & 0.0255 & $0.046 \mathrm{I}$ & 0.0379 & 0.0067 \\
\hline 9 & $\begin{array}{l}\text { operating } \\
\text { expenses to } \\
\text { revenue } \\
(\mathrm{OER})\end{array}$ & Independent & 30 & 0.2068 & 0.4409 & 0.3349 & 0.0599 & 30 & $0.283 \mathrm{I}$ & 0.5740 & 0.4189 & 0.0620 \\
\hline 10 & $\begin{array}{l}\text { asset } \\
\text { turnover } \\
\text { (ATO) }\end{array}$ & Independent & 30 & 0.0244 & 0.0860 & 0.0424 & 0.0153 & 30 & 0.0188 & 0.0424 & 0.0334 & 0.0068 \\
\hline II & $\begin{array}{l}\text { interest } \\
\text { income to } \\
\text { expenses } \\
\text { (IEE) }\end{array}$ & Independent & 30 & 0.0345 & 0.1097 & 0.0520 & 0.0219 & 30 & 0.0149 & 0.0557 & 0.0392 & 0.0118 \\
\hline $\mathrm{I} 2$ & $\begin{array}{l}\text { net non- } \\
\text { interest } \\
\text { margin } \\
(\text { NNIM) }\end{array}$ & Independent & 30 & $(0.0219)$ & 0.0056 & $(0.0023)$ & 0.0059 & 30 & $(0.0116)$ & 0.0140 & $(0.0027)$ & 0.0069 \\
\hline \multicolumn{13}{|c|}{ Asset-quality indicators } \\
\hline I3 & $\begin{array}{l}\text { provision } \\
\text { to earning } \\
\text { assets } \\
(\mathrm{PEA})\end{array}$ & Dependent & 30 & 0.0034 & 0.0629 & 0.0169 & $0.0 \mathrm{I} 4 \mathrm{I}$ & 30 & 0.0019 & 0.0333 & 0.0126 & 0.0080 \\
\hline $\mathrm{I} 4$ & $\begin{array}{l}\text { loan ratio } \\
\text { (LR) }\end{array}$ & Independent & 30 & 0.4745 & 0.7254 & 0.6227 & 0.0715 & 30 & 0.5096 & 0.7430 & 0.6275 & 0.0555 \\
\hline I5 & $\begin{array}{l}\text { loans to } \\
\text { deposits } \\
\text { (LTD) }\end{array}$ & Independent & 30 & 0.7842 & I.1693 & 0.9947 & 0.0879 & 30 & 0.7098 & 1.0788 & 0.9280 & 0.0890 \\
\hline \multicolumn{13}{|c|}{ Liquidity ratios } \\
\hline 16 & $\begin{array}{l}\text { cash to } \\
\text { assets } \\
\text { (CTA) }\end{array}$ & Dependent & 30 & 0.1187 & 0.3084 & 0.2045 & 0.0589 & 30 & 0.1049 & 0.4588 & 0.2410 & 0.1024 \\
\hline I7 & $\begin{array}{l}\text { cash to } \\
\text { deposits } \\
\text { (CTD) }\end{array}$ & Independent & 30 & 0.1816 & 0.5186 & $0.3 \mathrm{I} 35$ & 0.0973 & 30 & 0.1415 & 0.6813 & 0.3467 & 0.1708 \\
\hline \multicolumn{13}{|c|}{ Risk ratios } \\
\hline I8 & $\begin{array}{l}\text { equity } \\
\text { multiplier } \\
(\mathrm{EM})\end{array}$ & Dependent & 30 & 4.8842 & 8.5300 & 7.0133 & 1.0180 & 30 & 5.6693 & II.235I & 7.8136 & I.I864 \\
\hline
\end{tabular}




\begin{tabular}{|c|c|c|c|c|c|c|c|c|c|c|c|c|}
\hline I9 & $\begin{array}{l}\text { deposits to } \\
\text { assets } \\
\text { (DTA) }\end{array}$ & Independent & 30 & 0.5917 & 0.7175 & 0.6567 & 0.0399 & 30 & 0.5465 & 0.8408 & $0.7 \mathrm{I} 63$ & 0.0788 \\
\hline 20 & $\begin{array}{l}\text { equity to } \\
\text { deposits } \\
\text { (ETD) }\end{array}$ & Independent & 30 & 0.1733 & 0.2893 & 0.2226 & 0.0357 & 30 & 0.1074 & $0.3 \mathrm{I} 44$ & 0.1864 & 0.0444 \\
\hline $2 \mathrm{I}$ & $\begin{array}{l}\text { total } \\
\text { liabilities } \\
\text { to equity } \\
\text { (TLE) }\end{array}$ & Independent & 30 & 3.8842 & 7.5300 & 6.0133 & 1.0180 & 30 & 4.6693 & $\mathrm{I} 0.235 \mathrm{I}$ & 6.8136 & I.I864 \\
\hline 22 & $\begin{array}{l}\text { total } \\
\text { liabilities } \\
\text { to } \\
\text { shareholder } \\
\text { capital } \\
\text { (TLSC) }\end{array}$ & Independent & 30 & 3.8842 & 8.8818 & 6.6053 & 1.4566 & 30 & 4.6693 & I2.223I & 8.5448 & 1.8962 \\
\hline 23 & $\begin{array}{l}\text { retained } \\
\text { earnings to } \\
\text { total assets } \\
\text { (RETA) }\end{array}$ & Independent & 30 & 0 & 0.1504 & 0.0848 & 0.0423 & 30 & 0 & 0.0870 & 0.0298 & 0.0329 \\
\hline
\end{tabular}

For the dependent variables, the author has chosen ROA, NIM, PEA, CA and EM. The dependent variables correspond to studies made by Pradhan and Shrestha (2016) which stated that management efficiency has a positive strong correlation to the bank performance. While macro-economic factors are important, they do not play a vital role on the impact of the individual performance of the bank. The study further revealed that ROA and NIM could be decisive factors affecting banks performance. Menicucci and Paolucci (2016) further investigated the relation between bank specific factors with profitability in the European Banking Sector to ascertain the role of internal factors achieving higher profitability. His analysis used regression model for 35 banks based on capital ratios and loan loss provision ratios on the efficiency of the banks over the period 2009 to 2013. His study suggested that banks with higher deposits and loan ratios are more profitable but their effect was statistically insignificant to compare efficiency of these banks. Abobaker (2018) study stated that high profitability can be achieved by increasing the bank assets, capital ratio and operating income. Consequently, the profitability is reduced as non-interest income increases over time. Following the same concepts for conventional and Islamic banks as provided in Table 2 above, there is nominal deviation in ROA, NIM and Capital Adequacyof the conventional and Islamic banks in the UAE. The reason could include that banks in UAE apply similar interest rates and have sufficient liquid assets within UAE without considering macro-economic factors. There is significant variation in PEA and the reason could be the shariah requirements which need to be followed by the Islamic banks as these are more conservative in their lending practices.

For independent variables, the table reveals range of variation. Most notable variation is seen in ROE, ED, TLSC and RETA. The reason for large difference in the means is due to size and composition of the banks' balance sheet. Also, some banks have been in existence for a longer time than others viz conventional banks in the UAE. While other independent variables indicate insignificant variation, it signifies consistency of the business model between Islamic and conventional banks. It has to be known that UAE is an overbanked market with 22 local national banks, 27 branches of foreign banks and II wholesale foreign bank branches as at 30 June 20I9. Further, even though there is demand for Islamic products, UAE Islamic banks enjoy a relatively small market share as compared to its conventional counterparts as stated by study conducted by Kapur (2020).

\section{Findings and Analysis \\ 3.I Model I}

Hypothesis I:There is no significant difference between profitability ratios of conventional banks and Islamic banks in the UAE

Dependent Variable: Return on Assets (ROA)

Independent Variable: Return on Equity (ROE), Profit Margin (PM), Return on Deposits (RD), Net Operating Margin (NOM) 
The alternative hypothesis will be that there is significant difference between profitability ratios of conventional and Islamic banks in the UAE.

Table 3. Correlation matrix

\begin{tabular}{|c|c|c|c|c|c|c|c|c|c|c|c|}
\hline \multicolumn{6}{|c|}{ Conventional Banks } & \multicolumn{6}{|c|}{ Islamic Banks } \\
\hline & $\begin{array}{c}\mathrm{RO} \\
\mathrm{A}\end{array}$ & $\begin{array}{c}\mathrm{RO} \\
\mathrm{E}\end{array}$ & $\mathrm{PM}$ & $\begin{array}{c}\mathrm{RO} \\
\mathrm{D}\end{array}$ & NOM & & ROA & ROE & $\mathrm{PM}$ & ROD & $\begin{array}{c}\mathrm{NO} \\
\mathrm{M}\end{array}$ \\
\hline $\mathrm{ROA}$ & $\begin{array}{r}1.00 \\
0 \\
\end{array}$ & & & & & $\begin{array}{l}\mathrm{RO} \\
\mathrm{A}\end{array}$ & 1.000 & & & & \\
\hline ROE & $\begin{array}{r}0.84 \\
3\end{array}$ & $\begin{array}{r}1.00 \\
0\end{array}$ & & & & ROE & 0.922 & 1.000 & & & \\
\hline$\overline{\mathrm{PM}}$ & $\begin{array}{r}0.20 \\
9 \\
\end{array}$ & $\begin{array}{r}0.5 \mathrm{I} \\
4 \\
\end{array}$ & 1.000 & & & PM & $0.91 \mathrm{I}$ & $0.85 \mathrm{I}$ & 1.000 & & \\
\hline ROD & $\begin{array}{r}0.98 \\
2\end{array}$ & $\begin{array}{r}0.83 \\
5\end{array}$ & 0.273 & $\begin{array}{r}1.00 \\
0\end{array}$ & & $\begin{array}{l}\mathrm{RO} \\
\mathrm{D}\end{array}$ & 0.953 & 0.810 & 0.889 & 1.000 & \\
\hline NOM & $\begin{array}{r}0.3 \mathrm{I} \\
8\end{array}$ & $\begin{array}{r}0.00 \\
2\end{array}$ & 0.420 & $\begin{array}{r}0.30 \\
4 \\
\end{array}$ & 1.000 & $\begin{array}{l}\mathrm{NO} \\
\mathrm{M}\end{array}$ & $\begin{array}{r}- \\
0.090\end{array}$ & $\begin{array}{r}- \\
0.098\end{array}$ & $\begin{array}{r}- \\
0.098 \\
\end{array}$ & $\begin{array}{r}- \\
0.039 \\
\end{array}$ & $\begin{array}{r}1.00 \\
0\end{array}$ \\
\hline
\end{tabular}

Analysis of Table 3:

I) There is positive correlation between ROA and ROE for conventional and Islamic banks

2) There is positive correlation between ROA and PM for conventional and Islamic banks. While both show a positive relation, it is significantly stronger in Islamic banks

3) There is positive relation between ROA and ROD for conventional and Islamic banks

4) The correlation is mixed for ROA and NOM for conventional and Islamic banks. It is positive for conventional banks and negative for Islamic banks

Table 4. Regression analysis table

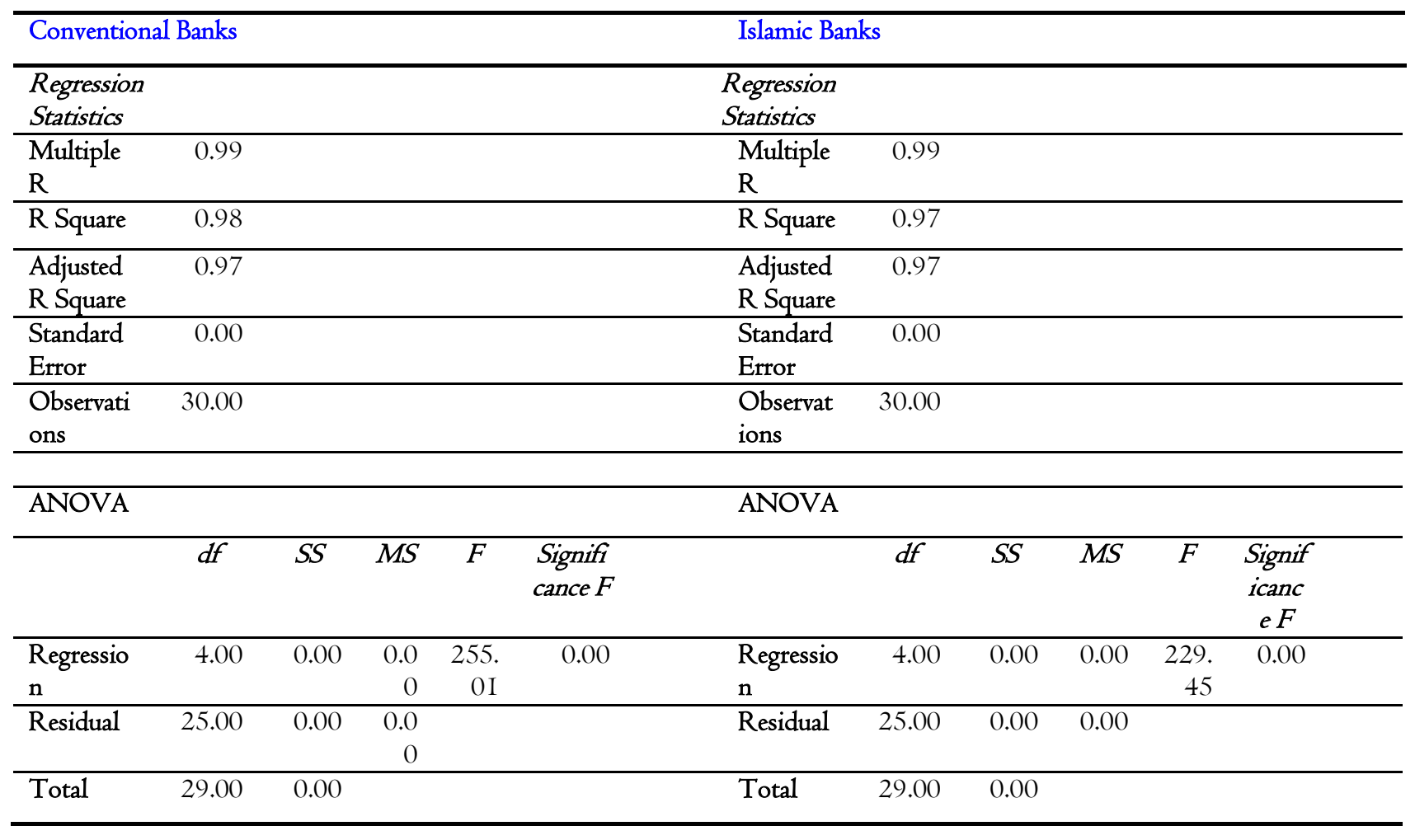




\begin{tabular}{|c|c|c|c|c|c|c|c|c|c|c|c|c|c|}
\hline & $\begin{array}{l}\text { Coeffi } \\
\text { cients }\end{array}$ & $\begin{array}{l}\text { Stand } \\
\text { ard } \\
\text { Error }\end{array}$ & $\begin{array}{c}t \\
\text { Stat }\end{array}$ & $\begin{array}{c}P- \\
\text { valu } \\
e\end{array}$ & $\begin{array}{c}\text { Lower } \\
95 \%\end{array}$ & $\begin{array}{c}\text { Uppe } \\
r \\
95 \%\end{array}$ & & $\begin{array}{l}\text { Coeffi } \\
\text { cients }\end{array}$ & $\begin{array}{l}\text { Stand } \\
\text { ard } \\
\text { Error }\end{array}$ & $t$ Stat & $\begin{array}{c}P_{-} \\
\text {value }\end{array}$ & $\begin{array}{c}\text { Lowe } \\
r \\
95 \%\end{array}$ & $\begin{array}{c}U_{P P} \\
e r \\
95 \\
\%\end{array}$ \\
\hline Intercept & $\begin{array}{r}0.001 \\
5\end{array}$ & 0.00 & $\begin{array}{r}0.6 \\
4\end{array}$ & 0.53 & -0.01 & 0.00 & Intercept & $\begin{array}{r}0.001 \\
8\end{array}$ & 0.00 & -0.13 & 0.90 & 0.00 & 0.00 \\
\hline $\begin{array}{l}\text { return on } \\
\text { equity } \\
\text { (ROE) }\end{array}$ & 0.04 & 0.02 & $\begin{array}{r}2.7 \\
9\end{array}$ & 0.01 & 0.01 & 0.07 & $\begin{array}{l}\text { return on } \\
\text { equity } \\
\text { (ROE) }\end{array}$ & 0.06 & 0.01 & 6.54 & 0.00 & 0.04 & 0.08 \\
\hline $\begin{array}{l}\text { profit } \\
\text { margin } \\
(\mathrm{PM})\end{array}$ & $-0.0 \mathrm{I}$ & 0.00 & $\begin{array}{r}2.8 \\
5\end{array}$ & 0.01 & $-0.0 \mathrm{I}$ & 0.00 & $\begin{array}{l}\text { profit } \\
\text { margin } \\
(\mathrm{PM})\end{array}$ & 0.00 & 0.00 & 0.48 & 0.64 & $-0.0 \mathrm{I}$ & 0.01 \\
\hline $\begin{array}{l}\text { return on } \\
\text { deposits } \\
\text { (ROD) }\end{array}$ & 0.59 & 0.05 & $\begin{array}{r}\mathrm{I} 2 . \\
5\end{array}$ & 0.00 & 0.49 & 0.69 & $\begin{array}{l}\text { return on } \\
\text { deposits } \\
\text { (ROD) }\end{array}$ & 0.42 & 0.05 & 7.88 & 0.00 & $0.3 \mathrm{I}$ & 0.54 \\
\hline $\begin{array}{l}\text { net } \\
\text { operating } \\
\text { margin } \\
(N O M)\end{array}$ & 0.00 & 0.00 & $\begin{array}{r}0.2 \\
3\end{array}$ & 0.82 & 0.00 & 0.00 & $\begin{array}{l}\text { net } \\
\text { operating } \\
\text { margin } \\
(\mathrm{NOM})\end{array}$ & 0.00 & 0.00 & -0.70 & 0.49 & 0.00 & 0.00 \\
\hline
\end{tabular}

Referring to Table 4, the adjusted $\mathrm{R}$ square value is 0.97 for both conventional and Islamic banks which means there is $97 \%$ variation in the dependent variables Return on Assets (ROA) is explained by independent variables Return on Equity (ROE), Profit Margin (PM), Return on Deposits (RD) and Net Operating Margin (NOM).

The value of F-stat is $255.0 \mathrm{I}$ for conventional banks and 229.45 for Islamic banks with df values $(4,25)$ and is significant as the level of significance is less than $5 \%$ or 0.05 , hence we can conclude that there is overall significant relationship between the predictor for independent variable Return on Equity (ROE), Profit Margin (PM), Return on Deposits (RD), Net Operating Margin (NOM) as a group and they predict the independent variable ROA significantly.

So, we reject the null hypothesis and accept the alternative hypothesis which indicate that significant difference between profitability ratios of conventional and Islamic banks in the UAE.

To assess the significance of each independent variable on the dependent variable ROA, it is established that ROE, $\mathrm{PM}$ and ROD have significant impact on ROA for conventional banks as their P value is less than 0.05 and similarly for Islamic banks ROE and ROD have significant impact. The NOM for conventional banks and PM, NOM for Islamic banks do not have any significance on ROA as its $\mathrm{P}$ value is greater than 5\%.

The following model can be created for conventional banks:

$\mathrm{ROA}=0.00 \mathrm{I} 5+0.04 \mathrm{ROE}-0.0 \mathrm{I} \mathrm{PM}+0.59 \mathrm{ROD}+0 \mathrm{NOM}+$ Error

The following model can be created for Islamic banks:

$\mathrm{ROA}=0.0018+0.06 \mathrm{ROE}+0 \mathrm{PM}+0.42 \mathrm{ROD}+0 \mathrm{NOM}+$ Error

\subsection{Model 2}

Hypothesis 2:There is no significant difference between efficiency ratios of the conventional banks and Islamic banks in the UAE

Dependent Variable:Net Interest Margin (NIM)

Independent Variable.Operating expense to Assets (OEA), Operating income to Assets (OIA), Operating Expenses to Revenue (OER), Asset Turnover (ATO), Interest Income to Expenses (IEE), Net Non-Interest Margin (NNIM)

The alternative hypothesis will be that there is significant difference between efficiency ratios of conventional and Islamic banks in the UAE. 
Table 5. Correlation Analysis

\begin{tabular}{|c|c|c|c|c|c|c|c|c|c|c|c|c|c|c|c|}
\hline \multicolumn{8}{|c|}{ Conventional Banks } & \multicolumn{8}{|c|}{ Islamic Banks } \\
\hline & $\overline{N I M}$ & $O E A$ & $O I A$ & $O E R$ & $\overline{A T O}$ & $I E E$ & $\begin{array}{c}N N I \\
M\end{array}$ & & $\overline{N I M}$ & $O E A$ & $O I A$ & $O E R$ & $\overline{A T O}$ & $I E E$ & $\begin{array}{c}N N I \\
M\end{array}$ \\
\hline NIM & 1.0000 & & & & & & & NIM & $\begin{array}{l}1.000 \\
0\end{array}$ & & & & & & \\
\hline OEA & 0.9315 & 1.0000 & & & & & & OEA & $\begin{array}{l}0.752 \\
7\end{array}$ & $\begin{array}{l}\text { I.000 } \\
0\end{array}$ & & & & & \\
\hline OIA & 0.9756 & $0.969 \mathrm{I}$ & 1.0000 & & & & & OIA & $\begin{array}{l}0.872 \\
7 \\
\end{array}$ & $\begin{array}{l}0.740 \\
8 \\
\end{array}$ & $\begin{array}{l}1.000 \\
0\end{array}$ & & & & \\
\hline OER & $0.4 \mathrm{I} 27$ & 0.6803 & 0.4910 & 1.0000 & & & & OER & $\begin{array}{l}(0.07 \\
6) \\
\end{array}$ & $\begin{array}{l}0.447 \\
\mathrm{I}\end{array}$ & $\begin{array}{l}(0.26 \\
0)\end{array}$ & $\begin{array}{l}.000 \\
0\end{array}$ & & & \\
\hline ATO & 0.9833 & $0.936 \mathrm{I}$ & $0.963 \mathrm{I}$ & 0.4658 & $\begin{array}{l}1.000 \\
0\end{array}$ & & & ATO & $\begin{array}{l}0.888 \\
0\end{array}$ & $\begin{array}{l}0.595 \\
7\end{array}$ & $\begin{array}{l}0.720 \\
7\end{array}$ & $\begin{array}{l}(0.05 \\
8)\end{array}$ & $\begin{array}{l}1.000 \\
0\end{array}$ & & \\
\hline IEE & 0.9740 & 0.9617 & 0.9834 & 0.5008 & $\begin{array}{l}0.977 \\
4\end{array}$ & $\begin{array}{l}1.000 \\
0\end{array}$ & & IEE & $\begin{array}{l}0.976 \\
4\end{array}$ & $\begin{array}{l}0.797 \\
0\end{array}$ & $\begin{array}{l}0.898 \\
0\end{array}$ & $\begin{array}{l}(0.04 \\
\text { I) }\end{array}$ & $\begin{array}{l}0.877 \\
5\end{array}$ & $\begin{array}{l}1.000 \\
0\end{array}$ & \\
\hline $\begin{array}{l}\text { NNI } \\
M\end{array}$ & $\begin{array}{l}(0.8614 \\
)\end{array}$ & $\begin{array}{l}(0.794 \\
{ }^{(0.7}\end{array}$ & $\begin{array}{l}(0.764 \\
)\end{array}$ & $\begin{array}{l}(0.503 \\
)^{(25}\end{array}$ & $\begin{array}{l}(0.86 \\
\mathrm{I})\end{array}$ & $\begin{array}{l}(0.80 \\
2)\end{array}$ & 1.0000 & $\begin{array}{l}\mathrm{NNI} \\
\mathrm{M}\end{array}$ & $\begin{array}{l}(0.27 \\
9)\end{array}$ & $\begin{array}{l}(0.30 \\
9)\end{array}$ & $\begin{array}{l}\text { (0.II } \\
9)\end{array}$ & $\begin{array}{l}(0.25 \\
2)\end{array}$ & $\begin{array}{l}(0.18 \\
0)\end{array}$ & $\begin{array}{l}(0.27 \\
8)\end{array}$ & $\mathrm{I}$ \\
\hline
\end{tabular}

Correlation is significant at $\mathrm{p}$ value of 0.05 level

Analysis of Table 5:

I) There is positive correlation between NIM and OEA, OIA, ATO and IEE for conventional banks as well as Islamic banks. The correlation is positively strong for conventional banks as compared to Islamic banks.

2) There is negative correlation for NIM and NNIM for conventional and Islamic banks but it is strong negative in case of conventional banks. The correlation is weak for Islamic banks.

3) NIM is positively correlated with OER for conventional banks and negatively correlated for Islamic banks.

On an overall level there is positive correlation except for Net Non-Interest Margin Ratio for conventional and Islamic banks in the UAE.

Table 6. Regression analysis table

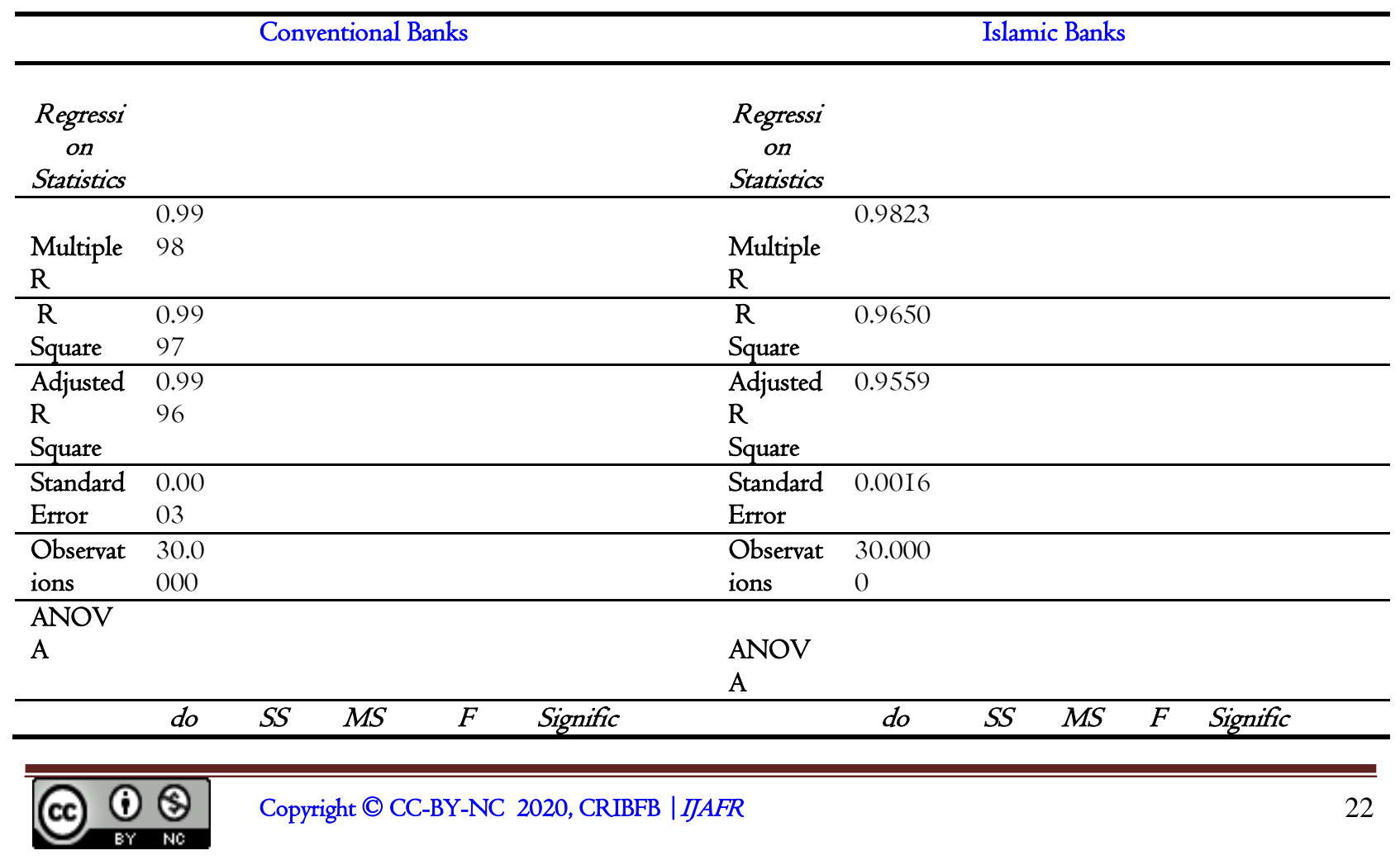




\begin{tabular}{|c|c|c|c|c|c|c|c|c|c|c|c|c|c|}
\hline & & & & & ance $F$ & & & & & & & ance $F$ & \\
\hline $\begin{array}{l}\text { Regressi } \\
\text { on }\end{array}$ & $\begin{array}{l}6.00 \\
00\end{array}$ & $\begin{array}{l}0.00 \\
74\end{array}$ & $\begin{array}{c}0.00 \\
12\end{array}$ & $\begin{array}{r}\text { I }, 532 . \\
6780\end{array}$ & 0.0000 & & $\begin{array}{l}\text { Regressi } \\
\text { on }\end{array}$ & 6.0000 & $\begin{array}{l}0.00 \\
16\end{array}$ & $\begin{array}{l}0.00 \\
03\end{array}$ & $\begin{array}{l}\mathrm{I05} \\
.67\end{array}$ & 0.0000 & \\
\hline Residual & $\begin{array}{l}23.0 \\
000\end{array}$ & $\begin{array}{l}0.00 \\
00\end{array}$ & $\begin{array}{l}0.00 \\
00\end{array}$ & & & & Residual & $\begin{array}{l}23.000 \\
0\end{array}$ & $\begin{array}{l}0.00 \\
01\end{array}$ & $\begin{array}{l}0.00 \\
00\end{array}$ & & & \\
\hline \multirow[t]{3}{*}{ Total } & $\begin{array}{l}29.0 \\
000\end{array}$ & $\begin{array}{l}0.00 \\
74\end{array}$ & & & & & Total & $\begin{array}{l}29.000 \\
0\end{array}$ & $\begin{array}{l}0.00 \\
16\end{array}$ & & & & \\
\hline & $\begin{array}{l}\text { Coef } \\
\text { ficie } \\
\text { nts }\end{array}$ & $\begin{array}{c}\text { Stan } \\
\text { dard } \\
\text { Error }\end{array}$ & $\begin{array}{c}t \\
\text { Stat }\end{array}$ & $P$-value & $\begin{array}{l}\text { Lower } \\
95 \%\end{array}$ & $\begin{array}{c}U_{P p} \\
e r \\
95 \\
\% \\
\end{array}$ & & $\begin{array}{c}\text { Coeffic } \\
\text { ients }\end{array}$ & $\begin{array}{l}\text { Stan } \\
\text { dard } \\
\text { Error }\end{array}$ & $\begin{array}{c}t \\
\text { Stat }\end{array}$ & $\begin{array}{c}P- \\
\text { valu } \\
e\end{array}$ & $\begin{array}{c}\text { Lower } \\
95 \%\end{array}$ & $\begin{array}{c}U_{P p} \\
\text { er } \\
95 \%\end{array}$ \\
\hline & 0.00 & 0.00 & 0.88 & 0.3855 & (0.00I & 0.00 & & 0.0280 & $0.0 \mathrm{I}$ & 1.82 & 0.0 & $(0.003$ & 0.05 \\
\hline Intercept & I3 & I5 & 46 & & 7) & 43 & Intercept & & 54 & 09 & 817 & 8) & 9 \\
\hline $\begin{array}{l}\text { Operatin } \\
\mathrm{g} \\
\text { expense } \\
\text { to assets } \\
\text { (OEA) }\end{array}$ & $\begin{array}{r}(0.8 \\
126)\end{array}$ & $\begin{array}{r}0.09 \\
95\end{array}$ & $\begin{array}{r}(8.16 \\
91)\end{array}$ & 0.0000 & $\begin{array}{r}(1.018 \\
3)\end{array}$ & $\begin{array}{l}(0.6 \\
06)\end{array}$ & $\begin{array}{l}\text { Operatin } \\
\mathrm{g} \\
\text { expense } \\
\text { to assets } \\
\text { (OEA) }\end{array}$ & 1.9835 & $\begin{array}{r}.04 \\
96\end{array}$ & $\begin{array}{r}1.88 \\
98\end{array}$ & $\begin{array}{r}0.0 \\
715\end{array}$ & $\begin{array}{r}(0.187 \\
7)\end{array}$ & $\begin{array}{r}4.15 \\
4\end{array}$ \\
\hline $\begin{array}{l}\text { Operatin } \\
\mathrm{g} \text { income } \\
\text { to assets } \\
\text { (OIA) }\end{array}$ & $\begin{array}{r}0.87 \\
93\end{array}$ & $\begin{array}{r}0.03 \\
91\end{array}$ & $\begin{array}{r}22.4 \\
666\end{array}$ & 0.0000 & 0.7983 & $\begin{array}{r}0.96 \\
02\end{array}$ & $\begin{array}{l}\text { operatin } \\
\mathrm{g} \text { income } \\
\text { to assets } \\
\text { (OIA) }\end{array}$ & $\begin{array}{r}(0.82 I \\
7)\end{array}$ & $\begin{array}{r}0.45 \\
35\end{array}$ & $\begin{array}{l}\text { (I.8 } \\
\text { I I) }\end{array}$ & $\begin{array}{r}0.0 \\
831\end{array}$ & $\begin{array}{r}(1.759 \\
9)\end{array}$ & $\begin{array}{r}0.11 \\
6\end{array}$ \\
\hline $\begin{array}{l}\text { Operatin } \\
\mathrm{g} \\
\text { expenses } \\
\text { to } \\
\text { revenue } \\
\text { (OER) }\end{array}$ & $\begin{array}{r}(0.0 \\
048)\end{array}$ & $\begin{array}{r}0.00 \\
4 \mathrm{I}\end{array}$ & $\begin{array}{r}(1.18 \\
59)\end{array}$ & 0.2478 & $\begin{array}{r}(0.013 \\
3)\end{array}$ & $\begin{array}{r}0.00 \\
36\end{array}$ & $\begin{array}{l}\text { Operatin } \\
\mathrm{g} \\
\text { expenses } \\
\text { to } \\
\text { revenue } \\
\text { (OER) }\end{array}$ & $\begin{array}{r}(0.073 \\
\mathrm{I})\end{array}$ & $\begin{array}{r}0.03 \\
65\end{array}$ & $\begin{array}{l}(2.0 \\
04)\end{array}$ & $\begin{array}{r}0.0 \\
569\end{array}$ & $\begin{array}{r}(0.148 \\
5)\end{array}$ & $\begin{array}{r}0.00 \\
2\end{array}$ \\
\hline $\begin{array}{l}\text { Asset } \\
\text { turnover } \\
\text { (ATO) }\end{array}$ & $\begin{array}{r}0.04 \\
16\end{array}$ & $\begin{array}{r}0.02 \\
66\end{array}$ & $\begin{array}{r}.56 \\
07\end{array}$ & 0.1323 & $\begin{array}{r}(0.013 \\
5)\end{array}$ & $\begin{array}{r}0.09 \\
66\end{array}$ & $\begin{array}{l}\text { Asset } \\
\text { turnover } \\
\text { (ATO) }\end{array}$ & 0.2699 & $\begin{array}{r}0.1 \mathrm{I} \\
77\end{array}$ & $\begin{array}{r}2.29 \\
35\end{array}$ & $\begin{array}{r}0.0 \\
313\end{array}$ & 0.0265 & $\begin{array}{r}0.5 \mathrm{I} \\
3\end{array}$ \\
\hline $\begin{array}{l}\text { Interest } \\
\text { income } \\
\text { to } \\
\text { expenses } \\
\text { (IEE) }\end{array}$ & $\begin{array}{r}0.02 \\
09\end{array}$ & $\begin{array}{r}0.02 \\
04\end{array}$ & $\begin{array}{r}1.02 \\
64\end{array}$ & 0.3 I54 & $\begin{array}{r}(0.021 \\
3)\end{array}$ & $\begin{array}{r}0.06 \\
31\end{array}$ & $\begin{array}{l}\text { Interest } \\
\text { income } \\
\text { to } \\
\text { expenses } \\
\text { (IEE) }\end{array}$ & $0.455 \mathrm{I}$ & $\begin{array}{r}0.1 \mathrm{I} \\
29\end{array}$ & $\begin{array}{r}4.03 \\
01\end{array}$ & $\begin{array}{r}0.0 \\
005\end{array}$ & 0.2215 & $\begin{array}{r}0.68 \\
8\end{array}$ \\
\hline $\begin{array}{l}\text { Net } \\
\text { non- } \\
\text { interest } \\
\text { margin } \\
\text { (NNIM } \\
\text { ) }\end{array}$ & $\begin{array}{r}(0.8 \\
885)\end{array}$ & $\begin{array}{r}0.02 \\
68\end{array}$ & $\begin{array}{r}(33.1 \\
63)\end{array}$ & 0.0000 & $\begin{array}{r}(0.943 \\
9)\end{array}$ & $\begin{array}{r}(0.8 \\
330 \\
)\end{array}$ & $\begin{array}{l}\text { Net } \\
\text { non- } \\
\text { interest } \\
\text { margin } \\
\text { (NNIM } \\
\text { ) }\end{array}$ & $\begin{array}{r}(0.015 \\
4)\end{array}$ & $\begin{array}{r}0.04 \\
91\end{array}$ & $\begin{array}{r}(0.3 \\
14)\end{array}$ & $\begin{array}{r}0.7 \\
563\end{array}$ & $\begin{array}{r}\text { (0.II7 } \\
\text { I) }\end{array}$ & $\begin{array}{r}0.08 \\
62\end{array}$ \\
\hline
\end{tabular}

Referring to Table 6, the adjusted R-Square for conventional banks is marginally higher than Islamic banks indicating higher variation of dependent variable Net Interest Margin (NIM) on independent variables Operating expense to Assets (OEA), Operating income to Assets (OIA), Operating Expenses to Revenue (OER), Asset Turnover (ATO), Interest Income to Expenses (IEE), Net Non-Interest Margin (NNIM). The variation for conventional banks is $99.9 \%$ whereas for Islamic banks it is at $95.59 \%$.

The value of F-stat is II,532.6 for conventional banks and I05.67 for Islamic banks with df values $(6,23)$ and is significant as the level of significance is less than $5 \%$ or 0.05 , hence we can conclude that there is overall significant relationship between the predictor for independent variable Operating expense to Assets (OEA), Operating income to Assets (OIA), 
Operating Expenses to Revenue (OER), Asset Turnover (ATO), Interest Income to Expenses (IEE), Net Non-Interest Margin (NNIM) as a group and they predict the dependent variable NIM significantly.

So, we reject the null hypothesis and accept the alternative hypothesis which indicate that there is significant difference between efficiency ratios of conventional and Islamic banks in the UAE.

To assess the significance of each independent variable on the dependent variable NIM, it is established that OEA, OIA and NNIM have significant impact on NIM for conventional banks as their P value is less than 0.05 and similarly for Islamic banks ATO and IEE have significant impact. The OER, ATO and IEE for conventional banks and OEA, OIA, OER, NNIM for Islamic banks do not have any significance on ROA as its $\mathrm{P}$ value is greater than $5 \%$.

The following model can be created for conventional banks:

$\mathrm{NIM}=0.0013-0.8 \mathrm{I} 26 \mathrm{OEA}+$ 0.8793OIA- 0.0048OER + 0.04I6ATO + 0.0209 IEE - 0.885 NNIM + Error

The following model can be created for Islamic banks:

$\mathrm{NIM}=0.0280+\mathrm{I} .9835 \mathrm{OEA}-0.82 \mathrm{I} 7 \mathrm{OIA}-0.073 \mathrm{I}$ OER + 0.2699 ATO + 0.455I IEE - 0.0I54 NNIM + Error

\subsection{Model 3}

Hypothesis 3: There is no significant difference between asset quality of the conventional banks and Islamic banks in the UAE.

Dependent Variable:Provision to Earning Assets (PEA)

Independent Variable:Loan Ratio (LR), Loans to Deposits (LTD)

The alternative hypothesis will be that there is significant difference between asset quality of conventional and Islamic banks in the UAE.

Table 7. Correlation Analysis

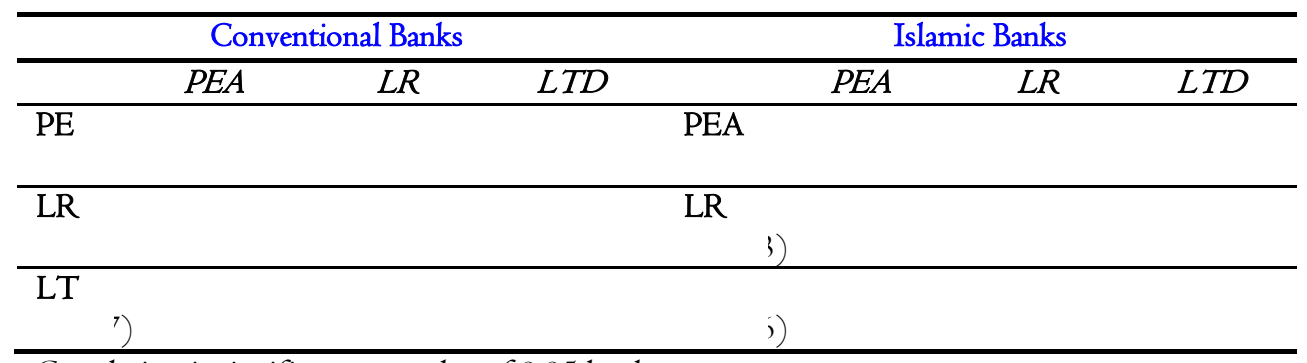

Correlation is significant at $\mathrm{p}$ value of 0.05 level

Analysis of Table 7:

I) There is weak positive correlation between Provision to earning Asset (PEA) and Loan Ratio (LR) for conventional banks and weak negative correlation for Islamic banks.

2) However, for loan to deposit ratio, both conventional and Islamic banks have a negative correlation between Provision to earning Asset (PEA) and Loan to Deposit (LTD)

The reason for mixed variation could be due to the lending restrictions under the sharia principles and the stringent loan criteria.

Table 8. Regression Analysis table

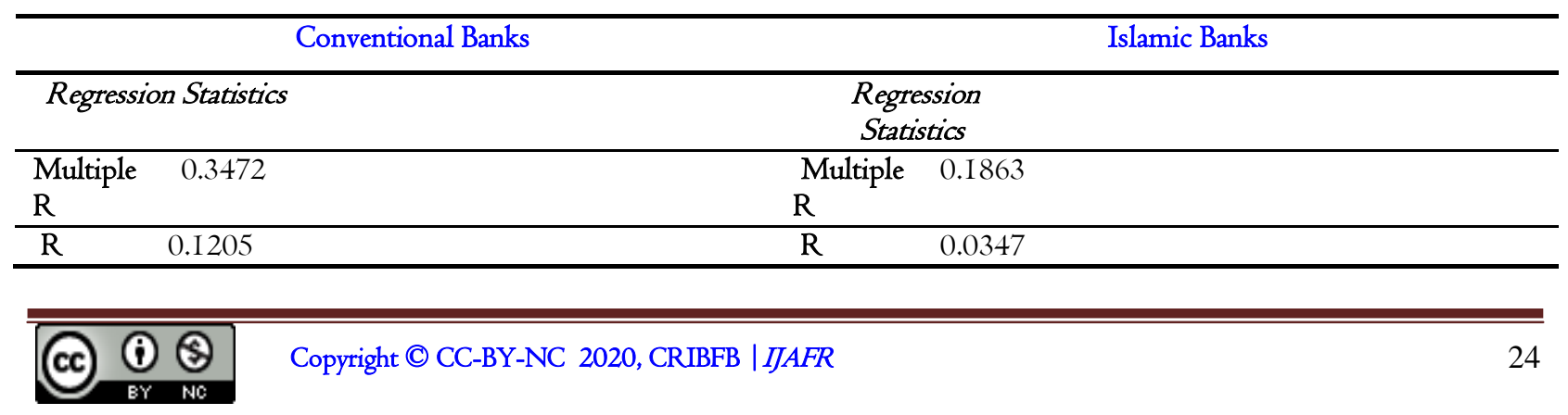




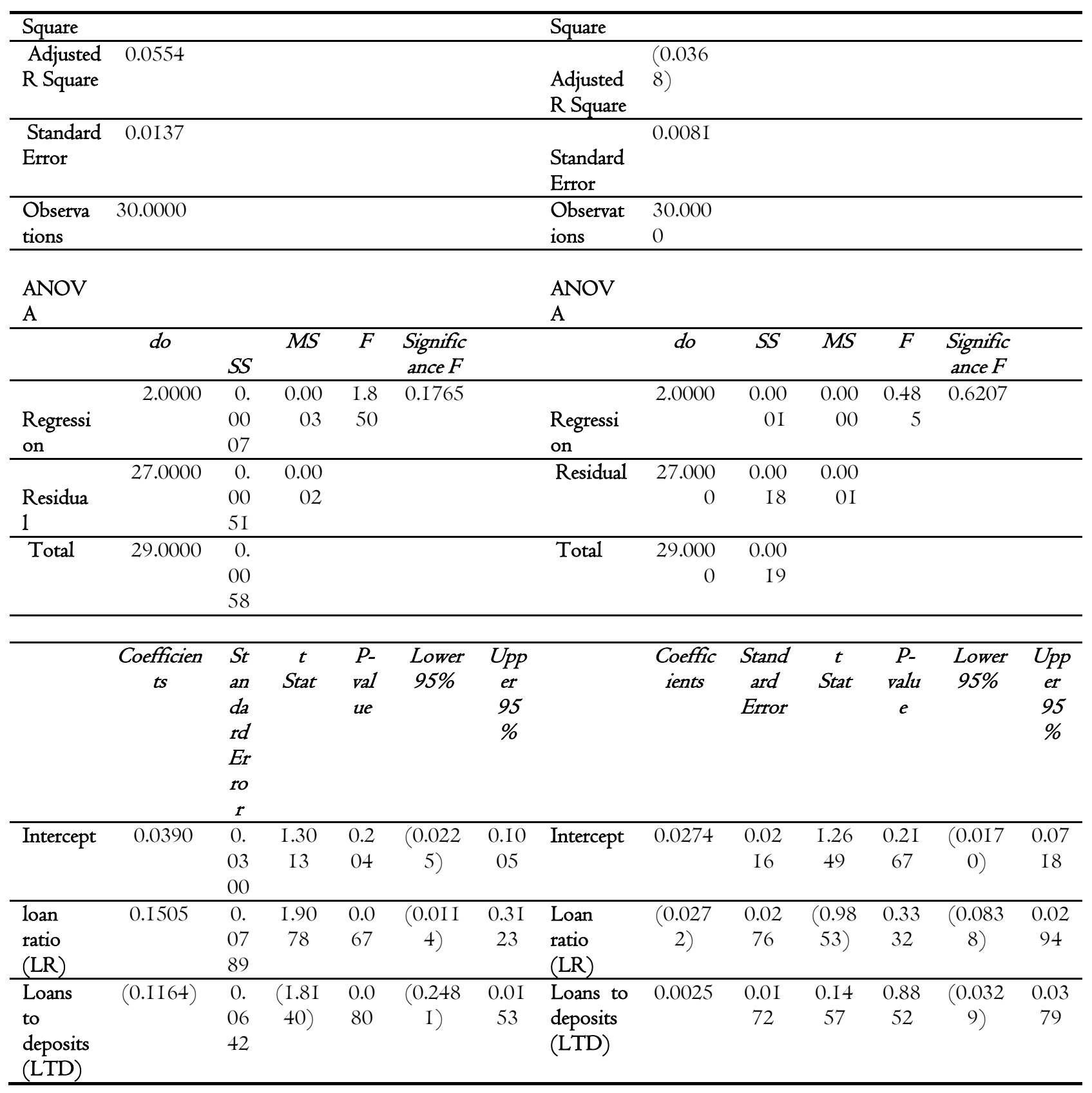

Referring to Table 8, the adjusted R-Square for conventional banks is significantly higher than Islamic banks indicating higher variation of dependent variable Provision to Earning Assets (PEA) on independent variables Loan Ratio (LR), Loans to Deposits (LTD). Infact the adjusted R Square for Islamic banks is negative indicating insignificance of exploratory values. The variation for conventional banks is $5.54 \%$ whereas for Islamic banks it is negative $3.68 \%$.

The value of F-stat is 1.85 for conventional banks and 0.485 for Islamic banks with dovalues $(2,27)$ and is significant as the level of significance is less than $5 \%$ or 0.05 , hence we can conclude that there is no significant relationship between the predictor for independent variable Loan Ratio (LR), Loans to Deposits (LTD) as a group and they do not predict the dependent variable PEA significantly.

So, we accept the null hypothesis and reject the alternative hypothesis which indicate that there is no significant difference between asset quality of the conventional banks and Islamic banks in the UAE.

To assess the significance of each independent variable on the dependent variable PEA, it is established that LR and LTDdo not have significant impact on PEA for conventional banks as well as Islamic banks as their P value is greater than 5\%. 
This proves that while the banks differ in profitability and efficiency, the asset quality structure and provisioning requirements have almost been similar since 2014 to 2018 .

The following model can be created for conventional banks:

PEA $=0.0390+0.1505$ LR-0.II64LTD + Error

The following model can be created for Islamic banks:

PEA $=0.0274$ I $0.0272 \mathrm{LR}+0.0025 \mathrm{LTD}+$ Error

\subsection{Model 4}

Hypothesis 4: There is no significant difference between Liquidity of the conventional and Islamic banks in the UAE

Dependent Variable:Cash to Assets (CTA)

Independent Variable:Cash to Deposits (CTD)

The alternative hypothesis will be that there is significant difference between liquidity of conventional and Islamic banks in the UAE.

Table 9. Correlation Analysis

\begin{tabular}{lccccc}
\hline \multicolumn{3}{c}{ Conventional Banks } & \multicolumn{3}{c}{ Islamic Banks } \\
\hline CTA & CTA & CTD & & CTA & CTD \\
\hline CTD & I.0000 & & CTA & I.0000 & I.0000 \\
& 0.9798 & CTD & 0.9582 & \\
\hline
\end{tabular}

The Correlation is significant at $\mathrm{p}$ value of 0.05 level. Accordingly, liquidity shows a strong positive correlation for conventional and Islamic banks in the UAE. The shows that both have managed to gather sufficient liquidity since 2014 to 2018 to fund the assets from the deposits by simultaneously maintaining liquid funds to meet unforeseen contingencies.

Table 10. Regression Analysis table

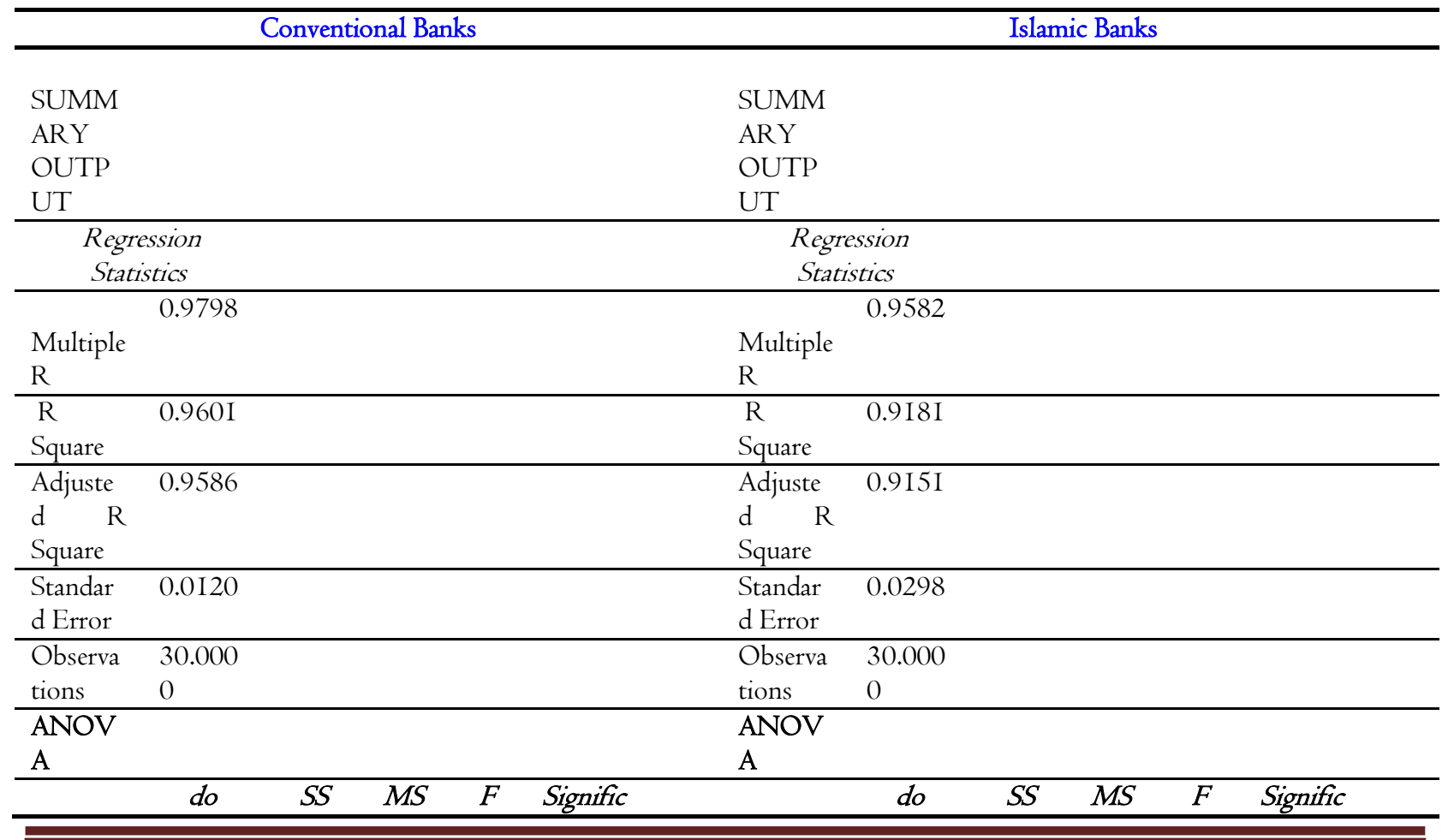




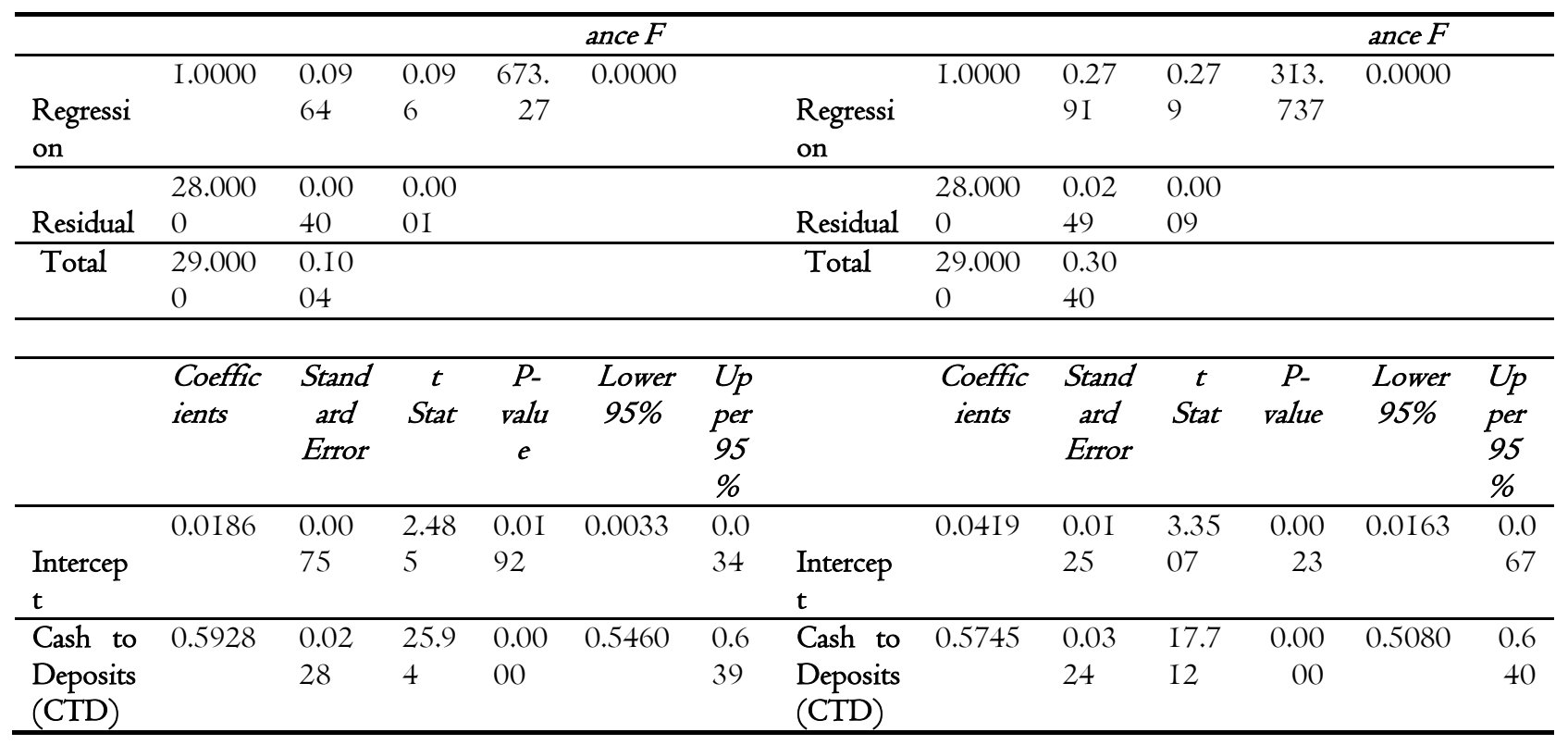

Referring to Table I0, the adjusted R-Square for conventional banks is marginally higher than Islamic banks indicating higher variation of dependent variable Cash to Asset (CTA) on independent variable Cash to Deposit (CTD). The variation for conventional banks is $95.8 \%$ whereas for Islamic banks it is at $91.51 \%$.

The value of F-stat is 673.2 for conventional banks and 313.737 for Islamic banks with do values $(\mathrm{I}, 28)$ and is significant as the level of significance is less than $5 \%$ or 0.05 , hence we can conclude that there is overall significant relationship between the predictor for independent variable Cash To Deposits (CTD) and it predicts the dependent variable Cash To Assets (CTA) significantly.

So, we reject the null hypothesis and accept the alternative hypothesis which indicate that there is significant difference between liquidity of conventional and Islamic banks in the UAE.

To assess the significance of each independent variable on the dependent variable CTA, it is established that CTD has significant impact on CTA for conventional and Islamic banks as their P value is less than 5\%.

The following model can be created for conventional banks:

$\mathrm{CTA}=0.0186+0.5928 \mathrm{CTD}+$ Error

The following model can be created for Islamic banks:

$\mathrm{CTA}=0.0419+0.5745 \mathrm{CTD}+$ Error

\subsection{Model 5}

Hypothesis 5: There is no significant difference between liquidity risk management of the conventional and Islamic banks in the UAE.

Dependent Variable:equity multiplier (EM)

Independent Variable:Deposits to Assets (DTA), Equity to Deposits (ETD), Total Liabilities to Equity (TLE), Total Liabilities to Shareholder Capital (TLSC), Retained Earnings to Total Assets (RETA)

The alternative hypothesis will be that there is significant difference between liquidity risk management of conventional and Islamic banks in the UAE.

Table II. Correlation Analysis

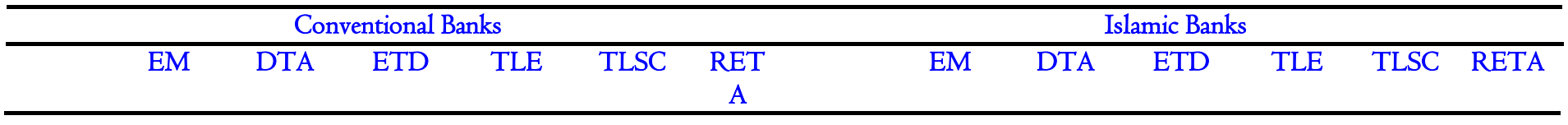




\begin{tabular}{|c|c|c|c|c|c|c|c|c|c|c|c|c|c|}
\hline EM & I.0000 & & & & & & EM & 1.0000 & & & & & \\
\hline DTA & $0.003 \mathrm{I}$ & I.0000 & & & & & DTA & 0.4547 & 1.0000 & & & & \\
\hline ETD & $\begin{array}{r}(0.9378) \\
)\end{array}$ & $\begin{array}{r}(0.332 \\
0)\end{array}$ & 1.0000 & & & & ETD & $\begin{array}{r}(0.82 \mathrm{I} \\
8)\end{array}$ & $\begin{array}{r}(0.837 \\
5)\end{array}$ & I.0000 & & & \\
\hline TEL & 1.0000 & $0.003 \mathrm{I}$ & $\begin{array}{r}(0.937 \\
8)\end{array}$ & I.0000 & & & TEL & 1.0000 & 0.4547 & $\begin{array}{r}(0.82 \mathrm{I} \\
8)\end{array}$ & I.0000 & & \\
\hline TLSC & 0.9540 & $\begin{array}{r}(0.030 \\
6) \\
\end{array}$ & $\begin{array}{r}(0.879 \\
2)\end{array}$ & 0.9540 & 1.0000 & & TLSC & $0.369 \mathrm{I}$ & 0.7258 & $\begin{array}{r}(0.668 \\
4) \\
\end{array}$ & $0.369 \mathrm{I}$ & $\begin{array}{r}.000 \\
0 \\
\end{array}$ & \\
\hline RET & $(0.4208$ & $(0.215$ & 0.4403 & $(0.420$ & $(0.486$ & 1.000 & RETA & $(0.408$ & 0.1116 & 0.1275 & $(0.408$ & 0.202 & 1.000 \\
\hline A & & 3) & & 8) & 2) & 0 & & 8) & & & 8) & 5 & 0 \\
\hline
\end{tabular}

Correlation is significant at $\mathrm{p}$ value of 0.05 level

Analysis of Table II:

I) correlation is weak. There is perfect positive correlation between EM and TLE indicating change in Total Liabilities to Equity in same proportion to Equity Multiplier. There is negative weak correlation for RETA and negative strong correlation for ETD.

2) For Islamic banks, the results are very similar to conventional banks, indicating that movement of ratios for risk management is almost identical.

The results prove that conventional and Islamic banks utilize similar strategies for risk management.

Table 12. Regression Analysis table

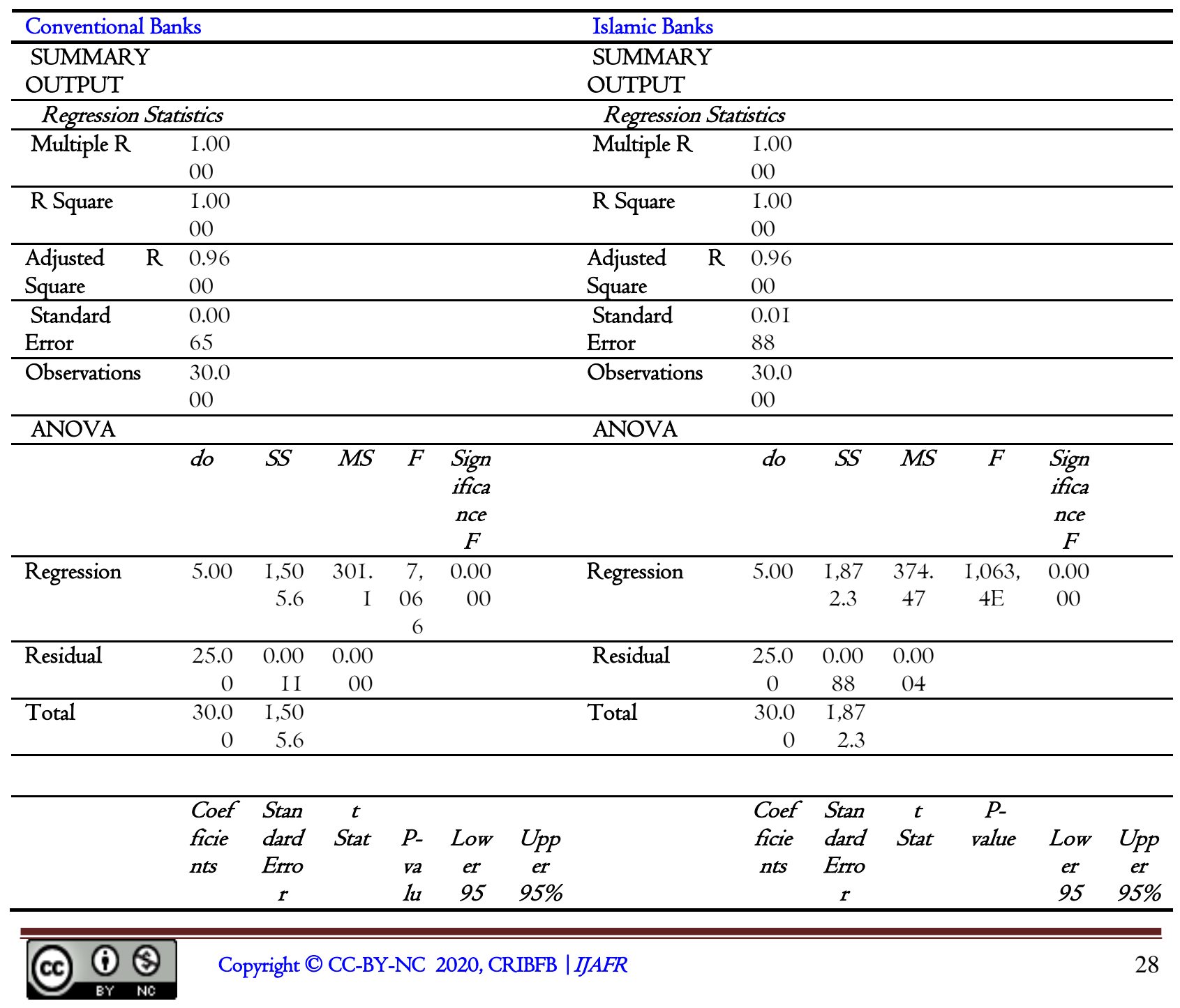




\begin{tabular}{|c|c|c|c|c|c|c|c|c|c|c|c|c|c|}
\hline & & & & $e$ & $\%$ & & & & & & & $\%$ & \\
\hline Intercept & 0 & $\begin{array}{r}\text { \#N } \\
/ \mathrm{A}\end{array}$ & $\begin{array}{r}\text { \#N } \\
/ \mathrm{A}\end{array}$ & $\begin{array}{r}\# \\
\text { N } \\
/ \\
\mathrm{A}\end{array}$ & $\begin{array}{r}\text { \#N } \\
/ \mathrm{A}\end{array}$ & $\begin{array}{r}\text { \#N } \\
/ \mathrm{A}\end{array}$ & Intercept & 0 & $\begin{array}{l}\text { \#N } \\
/ \mathrm{A}\end{array}$ & $\begin{array}{l}\text { \#N } \\
/ \mathrm{A}\end{array}$ & $\begin{array}{c}\# \mathrm{~N} / \\
\mathrm{A}\end{array}$ & $\begin{array}{c}\mathrm{N} \\
/ \mathrm{A}\end{array}$ & $\begin{array}{l}\mathrm{AN} \\
/ \mathrm{A}\end{array}$ \\
\hline $\begin{array}{l}\text { Deposits to } \\
\text { assets (DTA) }\end{array}$ & $\begin{array}{r}0.49 \\
10\end{array}$ & $\begin{array}{r}0.02 \\
18\end{array}$ & $\begin{array}{r}22.5 \\
33\end{array}$ & $\begin{array}{l}0 . \\
00 \\
00\end{array}$ & $\begin{array}{r}0.44 \\
62\end{array}$ & $\begin{array}{r}0.53 \\
59\end{array}$ & $\begin{array}{l}\text { Deposits to } \\
\text { assets (DTA) }\end{array}$ & $\begin{array}{r}0.55 \\
25\end{array}$ & $\begin{array}{r}0.05 \\
57\end{array}$ & $\begin{array}{r}9.9 \mathrm{I} \\
58\end{array}$ & $\begin{array}{r}0.000 \\
0\end{array}$ & $\begin{array}{r}0.43 \\
78\end{array}$ & $\begin{array}{r}0.66 \\
73\end{array}$ \\
\hline $\begin{array}{l}\text { Equity } \\
\text { deposits } \\
\text { (ETD) }\end{array}$ & $\begin{array}{r}.58 \\
09\end{array}$ & $\begin{array}{r}0.03 \\
88\end{array}$ & $\begin{array}{r}40.7 \\
17\end{array}$ & $\begin{array}{l}0 . \\
00 \\
00\end{array}$ & $\begin{array}{r}1.50 \\
10\end{array}$ & $\begin{array}{r}1.66 \\
09\end{array}$ & $\begin{array}{l}\text { Equity } \\
\text { deposits } \\
\text { (ETD) }\end{array}$ & $\begin{array}{r}1.73 \\
72\end{array}$ & $\begin{array}{r}0.06 \\
15\end{array}$ & $\begin{array}{r}28.2 \\
53\end{array}$ & $\begin{array}{r}0.000 \\
0\end{array}$ & $\begin{array}{r}1.6 \mathrm{I} \\
06\end{array}$ & $\begin{array}{r}1.86 \\
39\end{array}$ \\
\hline $\begin{array}{l}\text { Total liabilities } \\
\text { to equity } \\
\text { (TLE) }\end{array}$ & $\begin{array}{r}1.05 \\
39\end{array}$ & $\begin{array}{r}0.00 \\
42\end{array}$ & $\begin{array}{r}252 . \\
69\end{array}$ & $\begin{array}{r}0 . \\
00 \\
00\end{array}$ & $\begin{array}{r}1.04 \\
53\end{array}$ & $\begin{array}{r}1.06 \\
25\end{array}$ & $\begin{array}{l}\text { Total liabilities } \\
\text { to equity } \\
\text { (TLE) }\end{array}$ & $\begin{array}{r}1.03 \\
8 \mathrm{I}\end{array}$ & $\begin{array}{r}0.00 \\
38\end{array}$ & $\begin{array}{r}273 . \\
52\end{array}$ & $\begin{array}{r}0.000 \\
0\end{array}$ & $\begin{array}{r}1.03 \\
03\end{array}$ & $\begin{array}{r}1.04 \\
59\end{array}$ \\
\hline $\begin{array}{l}\text { Total liabilities } \\
\text { to shareholder } \\
\text { capital (TLSC) }\end{array}$ & $\begin{array}{r}(0.0 \\
6)\end{array}$ & $\begin{array}{r}0.00 \\
29\end{array}$ & $\begin{array}{r}(0.2 \\
\mathrm{I})\end{array}$ & $\begin{array}{r}0 . \\
83 \\
31\end{array}$ & $\begin{array}{r}(0.0 \\
0)\end{array}$ & $\begin{array}{r}0.00 \\
54\end{array}$ & $\begin{array}{l}\text { Total liabilities } \\
\text { to shareholder } \\
\text { capital (TLSC) }\end{array}$ & $\begin{array}{r}0.00 \\
21\end{array}$ & $\begin{array}{r}0.00 \\
28\end{array}$ & $\begin{array}{r}0.74 \\
72\end{array}$ & $\begin{array}{r}0.46 \mathrm{I} \\
9\end{array}$ & $\begin{array}{l}0.0 \\
03)\end{array}$ & $\begin{array}{r}0.00 \\
78\end{array}$ \\
\hline $\begin{array}{l}\text { Retained } \\
\text { earnings to } \\
\text { total assets } \\
\text { (RETA) }\end{array}$ & $\begin{array}{r}0.06 \\
08\end{array}$ & $\begin{array}{r}0.04 \\
19\end{array}$ & $\begin{array}{r}1.44 \\
9 \mathrm{I}\end{array}$ & $\begin{array}{r}0 . \\
15 \\
97\end{array}$ & $\begin{array}{l}(0.0 \\
25)\end{array}$ & $\begin{array}{r}0.14 \\
72\end{array}$ & $\begin{array}{l}\text { Retained } \\
\text { earnings to } \\
\text { total assets } \\
(\text { RETA) } \\
\end{array}$ & $\begin{array}{r}0.09 \\
87\end{array}$ & $\begin{array}{r}0.12 \\
83\end{array}$ & $\begin{array}{r}0.76 \\
9 \mathrm{I}\end{array}$ & $\begin{array}{r}0.449 \\
0\end{array}$ & $\begin{array}{r}(0.1 \\
6)\end{array}$ & $\begin{array}{r}0.36 \\
30\end{array}$ \\
\hline
\end{tabular}

Referring to Table 12, the adjusted R-Square for conventional banks and Islamic banks similar at 96\% indicating related variation of dependent variable equity multiplier (EM) on independent variables Deposits To Assets (DTA), Equity To Deposits (ETD), Total Liabilities to Equity (TLE), Total Liabilities to Shareholder Capital (TLSC), Retained Earnings to Total Assets (RETA).

The value of F-stat is 7,066 for conventional banks and I,063,4E for Islamic banks with do values $(5,25)$ and is significant as the level of significance is less than $5 \%$ or 0.05 , hence we can conclude that there is overall significant relationship between the predictor for independent variable Deposits To Assets (DTA), Equity To Deposits (ETD), Total Liabilities to Equity (TLE), Total Liabilities to Shareholder Capital (TLSC), Retained Earnings to Total Assets (RETA) as a group and they predict the dependent variable Equity Multiplier (EM) significantly.

So, we reject the null hypothesis and accept the alternative hypothesis which indicate that there is significant difference between liquidity risk management of conventional and Islamic banks in the UAE.

To assess the significance of each independent variable on the dependent variable EM, it is established that DTA, ETD and TLE have significant impact on EM for conventional banks as their P value is less than 0.05 and similarly for Islamic banks DTA, ETD and TLE have significant impact. The TLSC and RETA for conventional banks and Islamic banks do not have any significance on EM as its $\mathrm{P}$ value is greater than $5 \%$.

The following model can be created for conventional banks:

$\mathrm{EM}=0+0.4910$ DTA + I.5809 ETD + I.0539 TLE - 0.06 TLSC + 0.0608 RETA + Error

The following model can be created for Islamic banks:

$\mathrm{EM}=0+0.5525 \mathrm{DTA}+\mathrm{I} .7372 \mathrm{ETD}+\mathrm{I} .038 \mathrm{I}$ TLE - $0.06 \mathrm{TLSC}+0.0608 \mathrm{RETA}+$ Error

\section{Summary and Conclusion}

This paper examines the comparative performance between conventional and Islamic banks in the banks in UAE by using ratio analysis. The study uses several statistical tools to ensure their reliability, including mean testing, correlation and regression analysis. Results from the study indicate financial characteristics of profitability ratios, efficiency ratios, asset quality indicators and risk/ risk management ratios. The results clarify that Islamic banks are operationally efficient and profitable because of risks sharing and greater dependency on deposits capital. Overall, the ratios indicate, the conventional banks to have higher scores than the counterparts. Further, the statistical analysis of the comparative performance showed that the conventional and Islamic banks are significantly different for profitability, efficiency, liquidity, risk and risk management. However, there is no significant difference for asset quality ratios in the conventional and Islamic banks in the UAE. Islamic banks 
The reasons of variations can be attributed to several factors notably experience, size, governance, operational procedures, etc. These results pave way for the conventional and Islamic banks to re-engineer their strategic positions and consider decisions with deliberate care and effectives.

Considering the time limitation and busy schedules of the interviewees, the author was unable to conduct direct interview with the banks' management to judge the strategy of each bank under consideration, else the results could have been better interpreted for the internal performance of the bank. Further, an increased sample size taking into account GCC or MENA banks could have provided better understanding and comparison of conventional and Islamic banks in the region.

\section{References}

Abobaker, M. J. (20I8). Bank specific, industry concentration and macroeconomic determinants of Egyptian bank profitability. International Journal of Accounting and Financial Studies, 8(I), 380-397.

Al-Ajmi, J., Hussain, H. A., \& Al-Saleh, N. (2009). Clients of conventional and Islamic banks in Bahrain: How they choose which bank to patronize. International Journal of Social Economics, 36(II), I086-I II2.

Al-Hassan, A., Khamis, M., \& Oulidi, N. (2010). The GCC banking sector: Topography and analysis. Banks and Banks Systems, 5(3), I5-28.

Al-Muharrami, S., \& Matthews, K. (2009). Market power versus efficient-structure in Arab GCC banking. Working paper No.7, Cardiff business school.

Al-Tamimi, H. A., \& Al-Amiri, A. (2003). Analyzing service quality in the UAE Islamic banks. Journal of Financial Services Marketing, 8(2), I I9-I32

Bashier, B. A. (1983). Portfolio management of Islamic banks: A certainty approach. Journal of Banking and Finance, 7(3), 339-354.

Beck, T., Demirgüç-Kunt, A., \& Merrouche, O. (2010). Islamic vs. conventional banking: Business model, efficiency and stability. The World Bank.

Coats, P., \& Fant, L. (I993). Recognizing financial distress patterns using a neural network tool. Financial Management, 22(3), I42-I55.

Demirgue-Kunt, A., \& Hizinga, H. (I999). Determinants of commercial bank interest margins and profitability: Some international evidence. The World Bank Economic Review, I3(2), 379-408.

Essayyad, M., \& Madani, H. (2003). Investigating bank structure of an open petroleum economy: The case of Saudi Arabia. Managerial Finance, 29(II), 73-92.

Grais, W., \& Pellegrini, M. (2006). Corporate governance and Sharia compliance in institutions offering Islamic financial services. World Bank policy working paper: 4054, November, 2006 (pp. I-34).

Hanif, M., Tariq, M., \& Tahir, A. (2012). Comparative performance study of conventional and Islamic banking in Pakistan. International Research Journal of Finance \& Economics, (83).

Hume, J. (2004). Islamic finance: Provenance and prospects. International Financial Law Review, 23(5), I-4.

Hussain, M., Islam, M., Gunasekaran, A., \& Maskooki, K. (2002). Accounting standards and practices of financial institutions in GCC countries. Managerial Auditing Journal, I7(7), 350-362.

Iqbal, M. (2006). A broader definition of Riba: Pakistan Institute of Development Economics working paper. Institute of Islamic Banking and Insurance.

Islam, M. (2003). Development and performance of domestic and foreign banks in the GCC countries.

Kapur, M. (2020) Performance of Conventional and Islamic Banks in the UAE: A Comparative Graphical Ratio Analysis.

Mehta, A., \& Bhavani, G. (2017). What determines banks' profitability? evidence from emerging markets-the case of the UAE ban king sector. Accounting and Finance Research, 6(I), 77-88.

Menicucci, E., \& Paolucci, G. (2016). The determinants of bank profitability: empirical evidence from European banking sector. Journal of financial reporting and Accounting

Merchant, I. P. (2012). Empirical study of Islamic banks versus conventional banks of GCC. Global Journal of Management and Business Research, I2(20).

Pradhan, R. S., \& Shrestha, R. (2016). Impact of Bank Specific and Macroeconomic Variables on the Performance of Commercial Banks of Nepal. Available at SSRN 2793530

Rosly, S. A., \& Bakar, M. A. A. (2003). Performance of Islamic and mainstream banks in Malaysia. International Journal of Social Economics.

Annual Financial Statement of the Conventional and Islamic banks in the UAE

Abu Dhabi Commercial Bank (ADCB), 2014, 2015, 2016, 2017, 2018

Abu Dhabi Islamic Bank (ADIB), 2014, 2015, 2016, 2017, 2018

Al Hilal Bank (AHB), 20I4, 2015, 2016, 2017, 2018

Commercial Bank Dubai (CBD), 2014, 2015, 2016, 2017, 2018 
Dubai Islamic Bank (DIB), 20I4, 20I5, 2016, 2017, 2018

Emirates Islamic Bank (EIB), 20I4, 20I5, 2016, 2017, 2018

Emirates NBD (ENBD), 20I4, 2015, 2016, 2017, 2018

First Abu Dhabi Bank (FAB), 2016, 2017, 2018

First Gulf Bank (FGB), 20I4, 2015

Mashreq Bank, 2014, 2015, 2016, 2017, 2018

National Bank of Abu Dhabi (NBAD), 2014, 2015

Noor Bank (Noor), 20I4, 2015, 2016, 2017, 2018

RAK Bank (RAK), 2014, 2015, 2016, 2017, 2018

Sharjah Islamic Bank (SIB), 2014, 2015, 2016, 2017, 2018

\section{Copyrights}

Copyright for this article is retained by the author(s), with first publication rights granted to the journal. This is an open-access article distributed under the terms and conditions of the Creative Commons Attribution license (http://creativecommons.org/licenses/by/4.0/). 